Article

\title{
Variation Inequalities for One-Sided Singular Integrals and Related Commutators
}

\author{
Feng Liu ${ }^{1}$, Seongtae Jhang ${ }^{2}$, Sung-Kwun $\mathrm{Oh}^{3}$ and Zunwei Fu ${ }^{2,4, *}$ \\ 1 College of Mathematics and System Science, Shandong University of Science and Technology, \\ Qingdao 266590, China; FLiu@sdust.edu.cn \\ 2 Department of Computer Science, The University of Suwon, Wau-ri, Bongdam-eup, Hwaseong-si, \\ Gyeonggi-do 445-743, Korea; stjhang@suwon.ac.kr \\ 3 Department of Electrical Engineering, The University of Suwon, Wau-ri, Bongdam-eup, Hwaseong-si, \\ Gyeonggi-do 445-743, Korea; ohsk@suwon.ac.kr \\ 4 School of Mathematical Sciences, Qufu Normal University, Qufu 273000, China \\ * Correspondence: fuzunwei@eyou.com
}

Received: 26 August 2019; Accepted: 18 September 2019; Published: 20 September 2019

check for updates

\begin{abstract}
We establish one-sided weighted endpoint estimates for the $\varrho$-variation $\left(\begin{array}{lll}\varrho & >\end{array}\right)$ operators of one-sided singular integrals under certain priori assumption by applying one-sided Calderón-Zygmund argument. Using one-sided sharp maximal estimates, we further prove that the $\varrho$-variation operators of related commutators are bounded on one-sided weighted Lebesgue and Morrey spaces. In addition, we also show that these operators are bounded from one-sided weighted Morrey spaces to one-sided weighted Campanato spaces. As applications, we obtain some results for the $\lambda$-jump operators and the numbers of up-crossings. Our main results represent one-sided extensions of many previously known ones.
\end{abstract}

Keywords: $\varrho$-variation; one-sided singular integral; commutator; one-sided weighted Morrey space; one-sided weighted Campanato space

JEL Classification: 42B20; 42B25

\section{Introduction}

Given a family of bounded operators $\mathcal{T}=\left\{T_{\epsilon}\right\}_{\epsilon>0}$ acting between spaces of functions, one of the most significative problems in harmonic analysis is the existence of $\operatorname{limits}_{\lim } \rightarrow 0^{+} T_{\epsilon} f$ and $\lim _{\epsilon \rightarrow \infty} T_{\epsilon} f$, when $f$ belongs to a certain space of functions. The question that arises naturally is how to measure the speed of convergence of the above limits. A classic method is to investigate square functions of the type $\left(\sum_{i=1}^{\infty}\left|T_{\epsilon_{i}} f-T_{\epsilon_{i+1}} f\right|^{2}\right)^{1 / 2}$. Along this line, there is a more general way to study the following oscillation operator

$$
\mathcal{O}(\mathcal{T}) f(x)=\left(\sum_{i=1}^{\infty} \sup _{t_{i+1} \leq \epsilon_{i+1}<\epsilon_{i} \leq t_{i}}\left|T_{\epsilon_{i+1}} f(x)-T_{\epsilon_{i}} f(x)\right|^{2}\right)^{1 / 2}
$$

with $\left\{t_{i}\right\}$ being a fixed sequence decreasing to zero. However, beyond that, another typical method is to consider the $\varrho$-variation operator defined by

$$
\mathcal{V}_{\varrho}(\mathcal{T}) f(x)=\sup _{\left\{\epsilon_{i}\right\} \searrow 0}\left(\sum_{i=1}^{\infty}\left|T_{\epsilon_{i}} f(x)-T_{\epsilon_{i+1}} f(x)\right|^{\varrho}\right)^{1 / \varrho}
$$

where $\varrho>2$ and the supremum runs over all sequences $\left\{\epsilon_{i}\right\}$ of positive numbers decreasing to zero. 
The investigation on variation inequalities is an active research topic in probability, ergodic theory and harmonic analysis. The first variation inequality was proved by Lépingle [15] for martingales (also see [25] for a simple proof). Bourgain [2] proved the similar variation estimates for the ergodic averages of a dynamic system later. Bourgain's work has inspired a number of authors to investigate oscillation and variation inequalities for several families of operators from ergodic theory (see $[12,13,24]$ for examples) and harmonic analysis (cf. $[3,4,6,11,14])$. Recently, the variation inequalities and their weighed case for singular integrals and related operators have also been studied by many authors. The first work in this direction is due to Campbell et al. [3] who proved that $\mathcal{O}(\mathcal{H})$ and $\mathcal{V}_{\varrho}(\mathcal{H})$ with $\varrho>2$ are of type $(p, p)$ for $1<p<\infty$ and of weak type $(1,1)$, where $\mathcal{H}=\left\{H_{\epsilon}\right\}_{\epsilon>0}$ is the family of the truncated Hilbert transforms, i.e., $H_{\epsilon} f(x)=\int_{|x-y|>\epsilon} \frac{f(y)}{x-y} d y$. Subsequently, the aforementioned authors [4] also studied the variation operators related to the classical Riesz transform in $\mathbb{R}^{d}$ for $d \geq 2$. In 2004, Gillespie and Torrea [9] established the $L^{p}(\mathbb{R}, w(x) d x)$ bounds for $\mathcal{O}(\mathcal{H})$ and $\mathcal{V}_{\varrho}(\mathcal{H})$ with $\varrho>2$, $1<p<\infty$ and $w \in A_{p}$ (the Muckenhoupt weights class) (also see [10,14] for the related investigations). Later on, Crescimbeni et al. [5] proved that $\mathcal{O}(\mathcal{H})$ and $\mathcal{V}_{\varrho}(\mathcal{H})$ with $\rho>2$ map $L^{1}(\mathbb{R}, w(x) d x)$ into $L^{1, \infty}(\mathbb{R}, w(x) d x)$ for $w \in A_{1}$. In particular, Ma et al. [21,22] presented the weighted oscillation and variation inequalities for differential operators and Calderón-Zygmund singular integrals. Recently, Liu and $\mathrm{Wu}$ [19] established the weighted oscillation and variational inequalities for the commutator of one-dimensional Calderón-Zygmund singular integrals.

The primary purpose of this paper is to study weighted boundedness of oscillation and variational operators for one-sided singular integrals and their commutators. We say a function $K$ belongs to one-sided Calderón-Zygmund kernel OCZK $\left(B_{1}, B_{2}, B_{3}\right)$ if $K \in L_{\text {loc }}^{1}(\mathbb{R} \backslash\{0\})$ satisfies the following conditions: there exist constants $B_{1}, B_{2}, B_{3}>0$ such that

$$
\left|\int_{\{\epsilon<|x|<N\}} K(x) d x\right| \leq B_{1} \quad \text { for all } \epsilon \text { and all } N \text { with } 0<\epsilon<N,
$$

and furthermore $\lim _{\epsilon \rightarrow 0^{+}} \int_{\epsilon<|x|<N} K(x) d x$ exists,

$$
\begin{gathered}
|K(x)| \leq B_{2}|x|^{-1} \text { for all } x \neq 0 \\
|K(x-y)-K(x)| \leq B_{3}|y||x|^{-2} \text { for all } x \text { and } y \text { with }|x|>2|y|
\end{gathered}
$$

An example of a one-sided Calderón-Zygmund kernel is $K(x)=\frac{\sin (\log x)}{x \log x} \chi(0, \infty)$; see [1]. We mention here that the kernel of one-sided truncated Hilbert Transform, $K_{0}(x)=\frac{1}{x} \chi_{(0, \infty)}$, is not a OCZK for there does not exist a $B_{1}>0$ such that the first condition above holds.

Let $K \in \operatorname{OCZK}\left(B_{1}, B_{2}, B_{3}\right)$ with support in $(-\infty, 0)$ and $b \in \operatorname{BMO}(\mathbb{R})$. For $m \in \mathbb{N}$, we consider the one-sided operator

$$
T_{b}^{+, m} f(x)=\lim _{\epsilon \rightarrow 0^{+}} T_{\epsilon}^{+, b, m} f(x)=\text { p.v. } \int_{x}^{\infty}(b(x)-b(y))^{m} K(x-y) f(y) d y,
$$

where

$$
T_{\epsilon}^{+, b, m} f(x):=\int_{x+\epsilon}^{\infty}(b(x)-b(y))^{m} K(x-y) f(y) d y .
$$

For $m \geq 1$, the operator $T_{b}^{+, m}$ is the $m$-th order commutator of one-sided singular integral. When $m=0$, we denote by $T_{\epsilon}^{+, b, 0}=T_{\epsilon}^{+}$, and then the operator $T_{b}^{+, m}$ reduces to the one-sided Calderón-Zygmund singular integral operator $T^{+}$, which is defined by

$$
T^{+} f(x)=\lim _{\epsilon \rightarrow 0^{+}} T_{\epsilon}^{+} f(x)=\text { p.v. } \int_{x}^{\infty} K(x-y) f(y) d y .
$$


In 1997, Aimar et al. [1] observed that the operator $T^{+}$maps $L^{p}(\mathbb{R}, w(x) d x)$ into $L^{p}(\mathbb{R}, w(x) d x)$ for $1<p<\infty$ and $w \in A_{p}^{+}$, and maps $L^{1}(\mathbb{R}, w(x) d x)$ into $L^{1, \infty}(\mathbb{R}, w(x) d x)$ for $w \in A_{1}^{+}$. Subsequently, Lorente and Riveros [20] proved that there exist constants $C>0$ such that

$$
\left\|T_{b}^{+, m} f\right\|_{L^{p}(\mathbb{R}, w(x) d x)} \leq C\|b\|_{\mathrm{BMO}(\mathbb{R})}^{m}\|f\|_{L^{p}(\mathbb{R}, w(x) d x)}
$$

for $w \in A_{p}^{+}$and $1<p<\infty$, and

$$
w\left(\left\{x:\left|T_{b}^{+, m} f(x)\right|>\lambda\right\}\right) \leq C \phi_{m}\left(\|b\|_{\mathrm{BMO}(\mathbb{R})}^{m}\right) \int_{\mathbb{R}} \frac{|f(x)|}{\lambda}\left(1+\log ^{+}\left(\frac{|f(x)|}{\lambda}\right)\right)^{m} w(x) d x
$$

for $w \in A_{1}^{+}$and $\lambda>0$, where $\phi_{m}(t)=t\left(1+\log ^{+} t\right)^{m}$ and $z^{+}=\max \{z, 0\}$. Other interesting related results for the one-sided operators we may refer to [7,8,16-18], among others.

At first, we shall establish the one-sided weighted endpoint and strong estimates for the $\varrho$-variation $(\varrho>2)$ operators of one-sided singular integral and its commutator. Let us recall the one-sided weighted BMO spaces.

Definition 1. (One-sided weighted BMO spaces.) For a weight $w$, the one-sided weighted BMO spaces $\mathrm{BMO}^{+}(\mathbb{R}, w(x) d x)$ is defined by

$$
\mathrm{BMO}^{+}(\mathbb{R}, w(x) d x):=\left\{f \in L_{\text {loc }}^{1}(\mathbb{R}, d x):\|f\|_{\mathrm{BMO}^{+}(\mathbb{R}, w(x) d x)}:=\left\|M^{+, \sharp} f\right\|_{L^{\infty}(\mathbb{R}, w(x) d x)}<\infty\right\} .
$$

Here, $M^{+, \sharp}$ is one-sided sharp maximal operator defined by

$$
M^{+, \sharp} f(x)=\sup _{h>0} \frac{1}{h} \int_{x}^{x+h}\left(f(y)-\frac{1}{h} \int_{x+h}^{x+2 h} f(z) d z\right)^{+} d y .
$$

Remark 1. When $w(x) \equiv 1$, the space $\mathrm{BMO}^{+}(\mathbb{R}, w(x) d x)$ reduces to the one-sided $B M O$ space $\mathrm{BMO}^{+}(\mathbb{R})$, which was introduced by Martin-Reyes and de la Torre [23]. It was proved in [23] that

$$
M^{+, \sharp} f(x) \leq \sup _{h>0} \inf _{a \in \mathbb{R}}\left(\frac{1}{h} \int_{x}^{x+h}(f(y)-a)^{+} d y+\frac{1}{h} \int_{x+h}^{x+2 h}(a-f(y))^{+} d y\right) \leq\|f\|_{\mathrm{BMO}(\mathbb{R})}
$$

for any $x \in \mathbb{R}$. This yields that $\mathrm{BMO}(\mathbb{R}) \subset \mathrm{BMO}^{+}(\mathbb{R})$.

We now list our first main result as follows:

Theorem 1. Let $m \in \mathbb{N}, \varrho>2, b \in \mathrm{BMO}(\mathbb{R})$ and $K \in \operatorname{OCZK}\left(B_{1}, B_{2}, B_{3}\right)$ with supported in $(-\infty, 0)$. Let $\mathcal{T}_{b}^{m}=\left\{T_{\epsilon}^{+, b, m}\right\}_{\epsilon>0}$ and $\mathcal{T}=\left\{T_{\epsilon}^{+}\right\}_{\epsilon>0}$ be given as in Equation (1) and (2), respectively. Assume that $\left\|\mathcal{V}_{\varrho}(\mathcal{T})\right\|_{L^{q}(\mathbb{R}, d x) \rightarrow L^{q}(\mathbb{R}, d x)}<\infty$ for some $q \in(1, \infty)$. Then,

(i) for any $w \in A_{1}^{+}$and $f \in L^{1}(\mathbb{R}, w(x) d x)$, it holds that

$$
\left\|\mathcal{V}_{\varrho}(\mathcal{T}) f\right\|_{L^{1, \infty}(\mathbb{R}, w(x) d x)} \leq C\|f\|_{L^{1}(\mathbb{R}, w(x) d x)} ;
$$

(ii) for any $1<p<\infty, w \in A_{p}^{+}$and $f \in L^{p}(\mathbb{R}, w(x) d x)$, it holds that

$$
\left\|\mathcal{V}_{\varrho}\left(\mathcal{T}_{b}^{m}\right) f\right\|_{L^{p}(\mathbb{R}, w(x) d x)} \leq C\|b\|_{\mathrm{BMO}(\mathbb{R})}^{m}\|f\|_{L^{p}(\mathbb{R}, w(x) d x)} ;
$$

(iii) for a weight $w$ satisfying $w^{-1} \in A_{1}^{-}$and $f \in L^{\infty}(\mathbb{R}, w(x) d x)$, it holds that

$$
\left\|\mathcal{V}_{\varrho}(\mathcal{T}) f\right\|_{\mathrm{BMO}^{+}(\mathbb{R}, w(x) d x)} \leq C\|f\|_{L^{\infty}(\mathbb{R}, w(x) d x)} .
$$


In addition, we also investigate the boundedness behavior of the $\rho$-variation operators of one-sided singular integral and its commutator on one-sided weighted Morrey spaces and Companato spaces. In order to study the boundedness of one-sided singular integral operator on weighted Morrey spaces and Campanato spaces, Shi and Fu [27] introduced the one-sided weighted Morrey spaces and one-sided weighted Campanato spaces, which are defined as follows:

Definition 2. (One-sided weighted Morrey spaces and Campanato spaces.) Let $1 \leq p<\infty,-1 / p \leq$ $\beta<0$ and w be a weight on $\mathbb{R}$.

(i) One-sided weighted Morrey spaces $L^{p, \beta,+}(w)$ are defined by

$$
L^{p, \beta,+}(w):=\left\{f \in L_{\mathrm{loc}}^{p}(\mathbb{R}, d x):\|f\|_{L^{p, \beta,+}(w)}<+\infty\right\},
$$

where

$$
\|f\|_{L^{p, \beta,+}(w)}:=\sup _{x_{0} \in \mathbb{R}} \sup _{h>0} \frac{1}{h^{\beta}}\left(\frac{1}{w\left(\left(x_{0}-h, x_{0}\right)\right)} \int_{x_{0}}^{x_{0}+h}|f(x)|^{p} d x\right)^{1 / p} .
$$

(ii) One-sided weighted Campanato spaces $\mathfrak{L}^{p, \beta,+}(w)$ are given by

$$
\mathfrak{L}^{p, \beta,+}(w):=\left\{f \in L_{\mathrm{loc}}^{p}(\mathbb{R}, d x):\|f\|_{\mathfrak{L}^{p, \beta,+}(w)}<+\infty\right\},
$$

where

$$
\|f\|_{\mathcal{L}^{p, \beta,+}(w)}:=\sup _{x_{0} \in \mathbb{R}} \sup _{h>0} \frac{1}{h^{\beta}}\left(\frac{1}{w\left(\left(x_{0}-h, x_{0}\right)\right)} \int_{x_{0}}^{x_{0}+h}\left|f(x)-f_{\left(x_{0}, x_{0}+h\right)}\right|^{p} d x\right)^{1 / p} .
$$

Remark 2. It is well known that the following are valid:

$$
\begin{gathered}
\|f\|_{\mathfrak{L}^{p, \beta,+}(w)} \sim \sup _{x_{0} \in \mathbb{R}} \sup _{h>0} \inf _{a \in \mathbb{R}} \frac{1}{h^{\beta}}\left(\frac{1}{w\left(\left(x_{0}-h, x_{0}\right)\right)} \int_{x_{0}}^{x_{0}+h}|f(x)-a|^{p} d x\right)^{1 / p} ; \\
L^{p, \beta,+}(w) \subsetneq \mathfrak{L}^{p, \beta,+}(w) .
\end{gathered}
$$

The rest of the main results can be listed as follows.

Theorem 2. Let $m \in \mathbb{N}, \varrho>2, b \in \mathrm{BMO}(\mathbb{R})$ and $K \in \operatorname{OCZK}\left(B_{1}, B_{2}, B_{3}\right)$ with support in $(-\infty, 0)$. Let $\mathcal{T}_{b}^{m}=\left\{T_{\epsilon}^{+, b, m}\right\}_{\epsilon>0}$ and $\mathcal{T}=\left\{T_{\epsilon}^{+}\right\}_{\epsilon>0}$ be given as in Equation (1) and (2), respectively. Assume that $\left\|\mathcal{V}_{\varrho}(\mathcal{T})\right\|_{L^{q}(\mathbb{R}, d x) \rightarrow L^{q}(\mathbb{R}, d x)}<\infty$ for some $q \in(1, \infty)$. Then,

(i) for any $1<p<1 /(\beta+1),-1 / p \leq \beta<0, w \in A_{p}^{+}$and $f \in L^{p, \beta,+}(w)$,

$$
\left\|\mathcal{V}_{\varrho}\left(\mathcal{T}_{b}^{m}\right) f\right\|_{L^{p, \beta,+}(w)} \lesssim\|b\|_{\mathrm{BMO}(\mathbb{R})}^{m}\|f\|_{L^{p, \beta,+}(w)} ;
$$

(ii) for any $1<p<\infty,-1 / p \leq \beta<0, w \in A_{p}^{+}$and $f \in L^{p, \beta,+}(w)$,

$$
\left\|\mathcal{V}_{\varrho}(\mathcal{T}) f\right\|_{\mathfrak{L}^{p, \beta,+}(w)} \lesssim\|f\|_{L^{p, \beta,+}(w)} .
$$

Remark 3. We remark that we deal only with $\varrho>2$ for the variation operators in our main theorems, since it was pointed out in [2] that the variation is often not bounded in the case $\varrho \leq 2$. In addition, it is unknown what are the endpoint estimates of the variation operators for the commutators of one-sided singular integrals and whether the above operators are bounded from one-sided weighted Morrey spaces to one-sided weighted Campanato spaces, which are interesting. 
This paper is organized as follows. In Section 2, we shall present some basic definitions and necessary lemmas. In Section 3, we give the proofs of Theorems 1 and 2. As applications, we present the corresponding estimates for the $\lambda$-jump operators and the number of up-crossing for these operators in Section 4. Finally, some further comments will be given in Section 5. We would like to remark that our works and ideas are taken from $[9,19]$. It should also be pointed out that all results in this paper are valid for oscillation operator with similar arguments.

Throughout this paper, for any $p \in(1, \infty)$, we denote by $p^{\prime}$ the dual exponent to $p$, i.e., $1 / p+$ $1 / p^{\prime}=1$. The letter $C$ will represent a positive constant that may vary at each occurrence but is independent of the essential variables. For a weight $w$, an interval $I$ and a function $f: \mathbb{R} \rightarrow \mathbb{R}$, we denote by $w(I)=\int_{I} w(x) d x$ and $f_{I}=\frac{1}{|I|} \int_{I} f(x) d x$. We also use the convention $\sum_{i \in \varnothing} a_{i}=0$.

\section{Preliminaries}

We start with the definitions of one-sided Hardy-Littlewood maximal functions

$$
M^{+} f(x)=\sup _{h>0} \frac{1}{h} \int_{x}^{x+h}|f(y)| d y \text { and } M^{-} f(x)=\sup _{h>0} \frac{1}{h} \int_{x-h}^{x}|f(y)| d y .
$$

For $r>0$, we set $M_{r}^{+} f(x):=\left(M^{+}|f|^{r}(x)\right)^{1 / r}$.

By a weight, we mean a nonnegative measurable function.

Definition 3. [26] Let $1<p<\infty$. A weight $w$ belongs

to the class $A_{p}^{+}$(resp., $\left.A_{p}^{-}\right)$, if $[w]_{A_{p}^{+}}<\infty$ (resp., $\left.[w]_{A_{p}^{-}}<\infty\right)$, where

$$
\begin{aligned}
& {[w]_{A_{p}^{+}}:=\sup _{a<b<c} \frac{1}{(c-a)^{p}}\left(\int_{a}^{b} w(x) d x\right)\left(\int_{b}^{c} w(x)^{1-p^{\prime}} d x\right)^{p-1},} \\
& {[w]_{A_{p}^{-}}:=\sup _{a<b<c} \frac{1}{(c-a)^{p}}\left(\int_{b}^{c} w(x) d x\right)\left(\int_{a}^{b} w(x)^{1-p^{\prime}} d x\right)^{p-1} .}
\end{aligned}
$$

$A$ weight $w$ belongs to the class $A_{1}^{+}$(resp., $\left.A_{1}^{-}\right)$, if $[w]_{A_{1}^{+}}<\infty$ (resp., $\left.[w]_{A_{1}^{-}}<\infty\right)$, where

$$
[w]_{A_{1}^{+}}:=\sup _{x \in \mathbb{R}} w(x)^{-1} M^{-} w(x) \text { and }[w]_{A_{1}^{-}}:=\sup _{x \in \mathbb{R}} w(x)^{-1} M^{+} w(x) .
$$

Since the $A_{p}^{+}$and $A_{p}^{-}$classes are increasing with respect to $p$, the $A_{\infty}^{+}$(resp., $A_{\infty}^{-}$) class of weights is defined in a natural way by $A_{\infty}^{+}=\bigcup_{1<p<\infty} A_{p}^{+}$(resp., $\left.A_{\infty}^{-}=\bigcup_{1<p<\infty} A_{p}^{-}\right)$with

$$
[w]_{A_{\infty}^{+}}:=\inf _{1<p<\infty} \inf _{w \in A_{p}^{+}}[w]_{A_{p}^{+}}, \quad[w]_{A_{\infty}^{-}}:=\inf _{1<p<\infty} \inf _{w \in A_{p}^{-}}[w]_{A_{p}^{-}} .
$$

It is easy to see that $A_{p} \subsetneq A_{p}^{+}, A_{p} \subsetneq A_{p}^{-}$and $A_{p}=A_{p}^{+} \cap A_{p}^{-}$. Take $e^{x}$ for example, $e^{x} \notin A_{1}$, but $e^{x} \in A_{1}^{+}$. Here, $A_{p}$ denotes the usual Muckenhoupt weight.

It was shown in [26] that, for any $1<p<\infty, M^{+}: L^{p}(\mathbb{R}, w(x) d x) \rightarrow L^{p}(\mathbb{R}, w(x) d x)$ is bounded if and only if $w \in A_{p}^{+}$; moreover, $M^{+}: L^{1}(\mathbb{R}, w(x) d x) \rightarrow L^{1, \infty}(\mathbb{R}, w(x) d x)$ is bounded if and only if $w \in A_{1}^{+}$. The same results hold for $M^{-}$if $w \in A_{p}^{+}$replaced by $w \in A_{p}^{-}$for $1 \leq p<\infty$.

The following lemma will play key roles in our main proofs.

\section{Lemma 1.}

(i) Let $1 \leq p \leq \infty$ and $w \in A_{p}^{+}$. Then, for all $x_{0} \in \mathbb{R}$ and $h>0$,

$$
w\left(x_{0}-h, x_{0}+h\right) \leq\left(1+2^{p}[w]_{A_{p}^{+}}\right) w\left(x_{0}, x_{0}+h\right) .
$$


(ii) Let $1 \leq p \leq \infty$ and $w \in A_{p}^{+}$. Then, for all $x_{0} \in \mathbb{R}, h>0$ and $\lambda \geq 1$,

$$
w\left(x_{0}-\lambda h, x_{0}\right) \leq \lambda^{p}\left(2^{p}[w]_{A_{p}^{+}}+\left(2^{p}[w]_{A_{p}^{+}}\right)^{2}\right) w\left(x_{0}, x_{0}+h\right) .
$$

Proof. Fix $h>0$ and $x_{0} \in \mathbb{R}$ and we set $I=\left(x_{0}-h, x_{0}+h\right)$. Given two functions $f, g$ defined on $\mathbb{R}$, by Hölder's inequality, we get

$$
\begin{aligned}
& \left(\frac{1}{|I|} \int_{I}|f(x) g(x)| d x\right)^{p} \\
\leq & \frac{1}{|I|^{p}}\left(\int_{I}|f(x)|^{p} w(x) d x\right)\left(\int_{I}|g(x)|^{p^{\prime}} w(x)^{1-p^{\prime}} d x\right)^{p / p^{\prime}} \\
\leq & \left(\frac{1}{|I|} \int_{I^{-}} w(x) d x\right)\left(\frac{1}{|I|} \int_{I}|g(x)|^{p^{\prime}} w(x)^{1-p^{\prime}} d x\right)^{p-1}\left(\frac{1}{w\left(I^{-}\right)} \int_{I}|f(x)|^{p} w(x) d x\right) .
\end{aligned}
$$

Applying Equation (7) to the functions $f=\chi_{I^{+}}$and $g=\chi_{I^{+}}$, we get

$$
w\left(I^{-}\right) \leq 2^{p}[w]_{A_{p}^{+}} w\left(I^{+}\right) .
$$

Then, (5) follows easily from (8).

On the other hand, we get from (7) that

$$
\begin{aligned}
& \left(\frac{1}{|\lambda I|} \int_{\lambda I}|f(x) g(x)| d x\right)^{p} \\
\leq & \left(\frac{1}{|\lambda I|} \int_{(\lambda I)^{-}} w(x) d x\right)\left(\frac{1}{|\lambda I|} \int_{\lambda I}|g(x)|^{p^{\prime}} w(x)^{1-p^{\prime}} d x\right)^{p-1} \\
& \times\left(\frac{1}{w\left((\lambda I)^{-}\right)} \int_{\lambda I}|f(x)|^{p} w(x) d x\right) .
\end{aligned}
$$

Applying (9) to the functions $f=\chi_{I}$ and $g=\chi_{(\lambda I)^{+}}$, we have

$$
w\left((\lambda I)^{-}\right) \leq(2 \lambda)^{p}[w]_{A_{p}^{+}} w(I),
$$

which together with (5) yields (6).

By Lemma 2.1 in [26] and the similar argument as in classical Calderón-Zygmund decomposition for the usual Hardy-Littlewood maximal function, one can get the following Calderón-Zygmund decomposition for $\mathrm{M}^{+}$, which will be crucial for the proof of Lemma 3.

Lemma 2. Let $f \in L^{1}(\mathbb{R}, d x)$ and $\alpha>0$. Let $\Omega=\left\{x: M^{+} f(x)>\alpha\right\}$. Then, $\Omega$ can be decomposed into finitely many disjoint intervals of integers: $\Omega=\bigcup_{i} I_{i}$ with the following properties:

(i) $f=g+\varphi$, where $g=f \chi_{\mathbb{R} \backslash \Omega}$ and $g=f_{I_{i}}$ on $I_{i}$ for each $i$;

(ii) $\varphi=\sum_{i} \varphi_{i}$, where $\varphi_{i}=\left(f-f_{I_{i}}\right) \chi_{I_{i}}$;

(iii) $\|g\|_{L^{\infty}(\mathbb{R}, d x)} \leq 2 \alpha$ and $\|g\|_{L^{1}(\mathbb{R}, d x)} \leq\|f\|_{L^{1}(\mathbb{R}, d x)}$;

(iv) for each $i, \int_{I_{i}} \varphi_{i}(y) d y=0$ and $\frac{1}{\left|I_{i}\right|} \int_{I_{i}}\left|\varphi_{i}(y)\right| d y \leq 4 \alpha$;

(v) $\sum_{i}\left|I_{i}\right| \leq \alpha^{-1}\|f\|_{L^{1}(\mathbb{R}, d x)}$.

\section{Proofs of Main Results}

Following [9], let $\Theta=\left\{\beta: \beta=\left\{\epsilon_{i}\right\}, \epsilon_{i} \in \mathbb{R}, \epsilon_{i} \searrow 0\right\}$ and $F_{\varrho}$ be the mixed norm Banach space of two variables function $h$ defined on $\mathbb{N} \times \Theta$ such that

$$
\|h\|_{F_{\varrho}} \equiv \sup _{\beta}\left(\sum_{i}|h(i, \beta)|^{\varrho}\right)^{1 / \varrho}<\infty .
$$


Given a family of operators $\mathcal{T}=\left\{T_{t}\right\}_{t>0}$ defined on $L^{p}(\mathbb{R}, d x)$, we consider the $F_{Q^{-}}$-valued operator $V(\mathcal{T}): f \longrightarrow V(\mathcal{T}) f$ on $L^{p}(\mathbb{R}, d x)$ given by

$$
V(\mathcal{T}) f(x):=\left\{T_{\left[\epsilon_{i+1}, \epsilon_{i}\right]} f(x)\right\}_{\beta=\left\{\epsilon_{i}\right\} \in \Theta^{\prime}}
$$

where the expression $\left\{T_{\left[\epsilon_{i+1}, \epsilon_{i}\right]} f(x)\right\}_{\beta=\left\{\epsilon_{i}\right\} \in \Theta}$ is an abbreviation for the element of $F_{\varrho}$ given by

$$
(i, \beta)=\left(i,\left\{\epsilon_{i}\right\}\right) \longrightarrow T_{\left[\epsilon_{i+1}, \epsilon_{i}\right]} f(x):=T_{\epsilon_{i+1}} f(x)-T_{\epsilon_{i}} f(x) .
$$

Observe that

$$
\mathcal{V}_{\varrho}(\mathcal{T}) f(x)=\|V(\mathcal{T}) f(x)\|_{F_{\varrho^{\prime}}} \forall x \in \mathbb{R}
$$

In order to prove Theorem 1, we shall establish the following key result.

Lemma 3. Let $\varrho>2$ and $K \in \operatorname{OCZK}\left(B_{1}, B_{2}, B_{3}\right)$ with support in $(-\infty, 0)$. Let $\mathcal{T}=\left\{T_{\epsilon}^{+}\right\}_{\epsilon}>0$ be given as in Equation (2). Assume that $\left\|\mathcal{V}_{\varrho}(\mathcal{T})\right\|_{L^{q}(\mathbb{R}, w(x) d x) \rightarrow L^{q}(\mathbb{R}, w(x) d x)}<\infty$ for some $q \in(1, \infty)$ and $w \in A_{q}^{+}$. Then,

$$
\left\|\mathcal{V}_{\varrho}(\mathcal{T}) f\right\|_{L^{1, \infty}(\mathbb{R}, w(x) d x)} \leq C\|f\|_{L^{1}(\mathbb{R}, w(x) d x)}, \quad \forall f \in L^{1}(\mathbb{R}, w(x) d x) \text { and } w \in A_{1}^{+} .
$$

Proof. We shall adopt the classical Calderón-Zygmund argument to prove Lemma 3 . Let $\Omega=\left\{x: M^{+} f(x)>1\right\}$. Invoking Lemma 2, we can decompose $\Omega$ as $\Omega=\bigcup_{j} I_{j}$ and decompose $f$ as $f=g+\varphi$, where all $I_{j}$ are disjoint intervals, $g=f \chi_{\mathbb{R} \backslash \Omega}+\sum_{j} f_{I_{j}} \chi_{I_{j}}, \varphi=\sum_{j} \varphi_{j}, \varphi_{j}=\left(f-f_{I_{j}}\right) \chi_{I_{j^{\prime}}}$, $\|g\|_{L^{\infty}(\mathbb{R}, d x)} \leq 2,\|g\|_{L^{1}(\mathbb{R}, d x)} \leq\|f\|_{L^{1}(\mathbb{R}, d x)}$, and for each $j, \int_{I_{j}} \varphi_{j}(y) d y=0$ and $\frac{1}{\left|I_{j}\right|} \int_{I_{j}}\left|\varphi_{j}(y)\right| d y \leq 4$. It suffices to show that

$$
w\left(\left\{x: \mathcal{V}_{\varrho}(\mathcal{T}) f(x)>1\right\}\right) \leq C\|f\|_{L^{1}(\mathbb{R}, w(x) d x)} .
$$

It is clear that

$$
w\left(\left\{x: \mathcal{V}_{\varrho}(\mathcal{T}) f(x)>1\right\}\right) \leq w\left(\left\{x: \mathcal{V}_{\varrho}(\mathcal{T}) g(x)>1 / 2\right\}\right)+w\left(\left\{x: \mathcal{V}_{\varrho}(\mathcal{T}) \varphi(x)>1 / 2\right\}\right)
$$

By our assumption,

$$
\begin{aligned}
w\left(\left\{x: \mathcal{V}_{\varrho}(\mathcal{T}) g(x)>1 / 2\right\}\right) & \leq 2^{q} \int_{\mathbb{R}}\left|\mathcal{V}_{\varrho}(\mathcal{T}) g(x)\right|^{q} w(x) d x \\
& \leq C \int_{\mathbb{R}}|g(x)|^{q} w(x) d x \leq C\|f\|_{L^{1}(\mathbb{R}, w(x) d x)}
\end{aligned}
$$

We set $I_{j}=\left(c_{j}, c_{j}+\left|I_{j}\right|\right)$ and $\Omega^{*}=\bigcup_{j}\left(c_{j}-2\left|I_{j}\right|, c_{j}+2\left|I_{j}\right|\right)$, then

$$
w\left(\left\{x: \mathcal{V}_{\varrho}(\mathcal{T}) \varphi(x)>1 / 2\right\}\right) \leq w\left(\Omega^{*}\right)+w\left(\left\{x \in \mathbb{R} \backslash \Omega^{*}: \mathcal{V}_{\varrho}(\mathcal{T}) \varphi(x)>1 / 2\right\}\right) .
$$

Using Lemma 1 (i) and the $L^{1}(\mathbb{R}, w(x) d x) \rightarrow L^{1, \infty}(\mathbb{R}, w(x) d x)$ bounds for $M^{+}$, one has

$$
w\left(\Omega^{*}\right) \leq C \sum_{j} w\left(I_{j}\right)=C w(\Omega) \leq C\|f\|_{L^{1}(\mathbb{R}, w(x) d x)} .
$$

We now turn to prove

$$
w\left(\left\{x \in \mathbb{R} \backslash \Omega^{*}: \mathcal{V}_{\varrho}(\mathcal{T}) \varphi(x)>1 / 2\right\}\right) \leq C\|f\|_{L^{1}(\mathbb{R}, w(x) d x)} .
$$

For every $x \in \mathbb{R} \backslash \Omega^{*}$, we can choose a decreasing sequence $\left\{\epsilon_{i}\right\}$ (that depends on $x$ ) such that

$$
\mathcal{V}_{\varrho}(\mathcal{T}) \varphi(x) \leq 2\left(\sum_{i}\left|T_{\left[\epsilon_{i+1}, \epsilon_{i}\right]}^{+} \varphi(x)\right|^{\varrho}\right)^{1 / \varrho}
$$


For each $i$ and $x \in \mathbb{R} \backslash \Omega^{*}$, we set $B_{i}(x)=\left(x+\epsilon_{i+1}, x+\epsilon_{i}\right]$ and

$$
N_{i, 1}=\left\{j: I_{j} \subset B_{i}(x)\right\} \text { and } N_{i, 2}=\left\{j: I_{j} \cap B_{i}(x) \neq \varnothing, I_{j} \nsubseteq B_{i}(x)\right\}
$$

We notice that the cardinal of the $N_{i, 2}$ is at most two. Thus, it holds that

$$
\begin{aligned}
\mathcal{V}_{\varrho}(\mathcal{T}) \varphi(x) & \leq 2\left(\sum_{i}\left|\sum_{j \in N_{i, 1}} T_{\left[\epsilon_{i+1}, \epsilon_{i}\right]}^{+} \varphi_{j}(x)\right|^{\varrho}\right)^{1 / \varrho}+2\left(\sum_{i}\left|\sum_{j \in N_{i, 2}} T_{\left[\epsilon_{i+1}, \epsilon_{i}\right]}^{+} \varphi_{j}(x)\right|^{\varrho}\right)^{1 / \varrho} \\
& \leq 2 \sum_{i} \sum_{j \in N_{i, 1}}\left|T_{\left[\epsilon_{i+1}, \epsilon_{i}\right]}^{+} \varphi_{j}(x)\right|+4\left(\sum_{i} \sum_{j \in N_{i, 2}}\left|T_{\left[\epsilon_{i+1}, \epsilon_{i}\right]}^{+} \varphi_{j}(x)\right|^{\varrho}\right)^{1 / \varrho} .
\end{aligned}
$$

It follows that

$$
\begin{aligned}
& w\left(\left\{x \in \mathbb{R} \backslash \Omega^{*}: \mathcal{V}_{\varrho}(\mathcal{T}) \varphi(x)>1 / 2\right\}\right) \\
& \leq w\left(\left\{x \in \mathbb{R} \backslash \Omega^{*}: \sum_{i} \sum_{j \in N_{i, 1}}\left|T_{\left[\epsilon_{i+1}, \epsilon_{i}\right]}^{+} \varphi_{j}(x)\right|>\frac{1}{8}\right\}\right) \\
& +w\left(\left\{x \in \mathbb{R} \backslash \Omega^{*}:\left(\sum_{i} \sum_{j \in N_{i, 2}}\left|T_{\left[\epsilon_{i+1}, \epsilon_{i}\right]}^{+} \varphi_{j}(x)\right|^{\varrho}\right)^{1 / \varrho}>\frac{1}{16}\right\}\right) .
\end{aligned}
$$

Fix $x \in \mathbb{R} \backslash \Omega^{*}$. Note that $\left|x-c_{j}\right| \geq 2\left|I_{j}\right|>2\left|y-c_{j}\right|$ for any $y \in I_{j}$. Then, $\left|K(x-y)-K\left(x-c_{j}\right)\right| \leq$ $B_{3}\left|x-c_{j}\right|^{-2}\left|y-c_{j}\right|$. This together with the properties of $\varphi_{j}$ yield that

$$
\left|T_{\left[\epsilon_{i+1}, \epsilon_{i}\right]}^{+} \varphi_{j}(x)\right|=\left|\int_{\mathbb{R}}\left(K(x-y)-K\left(x-c_{j}\right)\right) \varphi_{j}(y) d y\right| \leq 2 B_{3}\left|I_{j}\right|\left|x-c_{j}\right|^{-2} \int_{I_{j}}|f(y)| d y .
$$

Observing that $T_{\left[\epsilon_{i+1}, \epsilon_{i}\right]}^{+} \varphi_{j}(x)=0$ if $x>c_{j}+\left|I_{j}\right|$, we thus have

$$
\begin{aligned}
& w\left(\left\{x \in \mathbb{R} \backslash \Omega^{*}: \sum_{i} \sum_{j \in N_{i, 1}}\left|T_{\left[\epsilon_{i+1}, \epsilon_{i}\right]}^{+} \varphi_{j}(x)\right|>\frac{1}{8}\right\}\right) \\
\leq & 8 \int_{\mathbb{R} \backslash \Omega^{*}} \sum_{i} \sum_{j \in N_{i, 1}}\left|T_{\left[\epsilon_{i+1}, \epsilon_{i}\right]}^{+} \varphi_{j}(x)\right| w(x) d x \\
\leq & 16 B_{3} \sum_{j}\left|I_{j}\right| \int_{\left(-\infty, c_{j}-2\left|I_{j}\right|\right]} \frac{w(x)}{\left|x-c_{j}\right|^{2}} d x \int_{I_{j}}|f(y)| d y .
\end{aligned}
$$

Fix $y \in I_{j}$. One can easily check that $c_{j}-x \geq 2(y-x) / 3$ for any $x \leq c_{j}-2\left|I_{j}\right|$. Then,

$$
\begin{aligned}
\int_{\left(-\infty, c_{j}-2\left|I_{j}\right|\right]} \frac{w(x)}{\left|x-c_{j}\right|^{\delta}} d x & \leq \sum_{k=1}^{\infty} \int_{\left[c_{j}-2^{k+1}\left|I_{j}\right|, c_{j}-2^{k}\left|I_{j}\right|\right]} \frac{w(x)}{\left|x-c_{j}\right|^{\delta}} d x \\
& \leq \sum_{k=1}^{\infty}\left(2^{k}\left|I_{j}\right|\right)^{-\delta} 2^{k+3}\left|I_{j}\right| \frac{1}{2^{k+3}\left|I_{j}\right|} \int_{y-2^{k+3}\left|I_{j}\right|}^{y} w(x) d x \\
& \leq C(\delta)\left|I_{j}\right|^{1-\delta} M^{-} w(y)
\end{aligned}
$$

for any $\delta>1$. By (19) and (20) (with $\delta=2)$ and $w \in A_{1}^{+}$, we have

$$
\begin{aligned}
& w\left(\left\{x \in \mathbb{R} \backslash \Omega^{*}: \sum_{i} \sum_{j \in N_{i, 1}}\left|T_{\left[\epsilon_{i+1}, \epsilon_{i}\right]}^{+} \varphi_{j}(x)\right|>\frac{1}{8}\right\}\right) \\
\leq & C \sum_{j} \int_{I_{j}}|f(y)| M^{-} w(y) d y \leq C\left([w]_{A_{1}^{+}}\right)\|f\|_{L^{1}(\mathbb{R}, w(x) d x)} .
\end{aligned}
$$


Fix $x \in \mathbb{R} \backslash \Omega^{*}$. Note that $T_{\left[\epsilon_{i+1}, \epsilon_{i}\right]}^{+} \varphi_{j}(x)=0$ when $x>c_{j}+\left|I_{j}\right|$. Moreover, $y-x \geq c_{j}-x \geq 0$ for any $y \in I_{j}$. Then,

$$
\left|T_{\left[\epsilon_{i+1}, \epsilon_{i}\right]}^{+} \varphi_{j}(x)\right| \leq B_{2} \int_{B_{i}(x)} \frac{\left|\varphi_{j}(y)\right|}{|x-y|} d y \leq B_{2}\left|x-c_{j}\right|^{-1} \chi_{\left(-\infty, c_{j}-2\left|I_{j}\right|\right]}(x) \int_{B_{i}(x)}\left|\varphi_{j}(y)\right| d y .
$$

Combining this with (20) (with $\delta=\varrho$ ) implies that

$$
\begin{aligned}
& w\left(\left\{x \in \mathbb{R} \backslash \Omega^{*}:\left(\sum_{i} \sum_{j \in N_{i, 2}}\left|T_{\left[\epsilon_{i+1}, \epsilon_{i}\right]}^{+} \varphi_{j}(x)\right|^{\varrho}\right)^{1 / \varrho}>\frac{1}{16}\right\}\right) \\
\leq & 16^{\varrho} \int_{\mathbb{R} \backslash \Omega^{*}} \sum_{i} \sum_{j \in N_{i, 2}}\left|T_{\left[\epsilon_{i+1}, \epsilon_{i}\right]}^{+} \varphi_{j}(x)\right|^{\varrho} w(x) d x \\
\leq & C(\varrho) \int_{\mathbb{R} \backslash \Omega^{*}} \sum_{j}\left(\sum_{i}\left|T_{\left[\epsilon_{i+1}, \epsilon_{i}\right]}^{+} \varphi_{j}(x)\right|\right)^{\varrho} w(x) d x \\
\leq & C(\varrho) \sum_{j} \int_{\left(-\infty, c_{j}-2\left|I_{j}\right|\right]}\left|x-c_{j}\right|^{-\varrho}\left(\sum_{i} \int_{B_{i}(x)}\left|\varphi_{j}(y)\right| d y\right)^{\varrho} w(x) d x \\
\leq & C(\varrho) \sum_{j} \int_{\left(-\infty, c_{j}-2\left|I_{j}\right|\right]} \frac{w(x)}{\left|x-c_{j}\right|^{\varrho}}\left(\int_{\mathbb{R}}\left|\varphi_{j}(y)\right| d y\right)^{\varrho} d x \\
\leq & C(\varrho) \sum_{j}\left|I_{j}\right|^{\varrho-1} \int_{I_{j}} \int_{\left(-\infty, c_{j}-2\left|I_{j}\right|\right]} \frac{w(x)}{\left|x-c_{j}\right|^{\varrho}} d x|f(y)| d y \\
\leq & C(\varrho) \sum_{j} \int_{I_{j}}|f(y)| M^{-}(w)(y) d y \\
\leq & C\left(\varrho,[w]_{A_{1}^{+}}\right)\|f\|_{L^{1}(\mathbb{R}, w(x) d x)^{\prime}}
\end{aligned}
$$

which together with (21) and (18) yields (17). Then, (12) follows from (13)-(17). This proves Lemma 3.

Applying similar arguments used in deriving Lemma 3, we can get the following:

Corollary 1. Let $K \in \operatorname{OCZK}\left(B_{1}, B_{2}, B_{3}\right)$ with support in $(-\infty, 0)$. Let $\varrho>2$ and $\mathcal{T}=\left\{T_{\epsilon}^{+}\right\}_{\epsilon>0}$ be given as in Equation (2). Assume that $\left\|\mathcal{V}_{\varrho}(\mathcal{T})\right\|_{L^{q}(\mathbb{R}, d x) \rightarrow L^{q}(\mathbb{R}, d x)}<\infty$ for some $q \in(1, \infty)$. Then,

$$
\left\|\mathcal{V}_{\varrho}(\mathcal{T}) f\right\|_{L^{1, \infty}(\mathbb{R}, d x)} \leq C\|f\|_{L^{1}(\mathbb{R}, d x)}, \quad \forall f \in L^{1}(\mathbb{R}, d x)
$$

The following lemma will play a pivotal role in the proof of Theorem 1.

Lemma 4. Let $m \in \mathbb{N}, \varrho>2, b \in \mathrm{BMO}(\mathbb{R})$ and $K \in \operatorname{OCZK}\left(B_{1}, B_{2}, B_{3}\right)$ with support in $(-\infty, 0)$. Let $\mathcal{T}_{b}^{m}=\left\{T_{\epsilon}^{+, b, m}\right\}_{\epsilon>0}$ and $\mathcal{T}=\left\{T_{\epsilon}^{+}\right\}_{\epsilon>0}$ be given as in Equations (1) and (2), respectively. Assume that $\left\|\mathcal{V}_{\varrho}(\mathcal{T})\right\|_{L^{q}(\mathbb{R}, d x) \rightarrow L^{q}(\mathbb{R}, d x)}<\infty$ for some $q \in(1, \infty)$. Then, for any $r>1$ and $x \in \mathbb{R}$, it holds that

$$
M^{+, \sharp}\left(\mathcal{V}_{\varrho}\left(\mathcal{T}_{b}^{m}\right) f\right)(x) \leq C\left(\sum_{i=0}^{m-1}\|b\|_{\mathrm{BMO}(\mathbb{R})}^{m-i} M_{r}^{+}\left(\mathcal{V}_{\varrho}\left(\mathcal{T}_{b}^{i}\right) f\right)(x)+\|b\|_{\mathrm{BMO}(\mathbb{R})}^{m} M_{r}^{+} f(x)\right) .
$$

Proof. We only prove (22) for the case $1<r<\min \{q, 2\}$, since $M_{r_{1}}^{+} f \leq M_{r_{2}}^{+} f$ for any $r_{2} \geq r_{1}$. Invoking Corollary 1 , we see that $\mathcal{V}_{\varrho}(\mathcal{T})$ is of weak type $(1,1)$. By the Marcinkiewicz interpolation theorem and our assumption, we have that $\mathcal{V}_{\varrho}(\mathcal{T})$ is bounded on $L^{p}(\mathbb{R}, d x)$ for any $1<p<q$. Fix $x_{0} \in \mathbb{R}$ and $h>0$. We decompose $f$ as $f=f_{1}+f_{2}+f_{3}$, where $f_{1}=f \chi_{\left[x_{0}, x_{0}+2 h\right]}$ and $f_{2}=f \chi_{\left(x_{0}+2 h, \infty\right)}$. Let $I=\left[x_{0}-2 h, x_{0}+2 h\right]$. In view of (3), to prove (22), we only prove 


$$
\begin{aligned}
& \frac{1}{h} \int_{x_{0}}^{x_{0}+h}\left|\mathcal{V}_{\varrho}\left(\mathcal{T}_{b}^{m}\right) f(y)-\mathcal{V}_{\varrho}\left(\mathcal{T}_{b}^{m}\right)\left(\left(b-b_{I}\right)^{m} f_{2}\right)\left(x_{0}\right)\right| d y \\
\leq & C\left(\sum_{i=0}^{m-1}\|b\|_{\mathrm{BMO}(\mathbb{R})}^{m-i} M_{r}^{+}\left(\mathcal{V}_{\varrho}\left(\mathcal{T}_{b}^{i}\right) f\right)(x)+\|b\|_{\mathrm{BMO}(\mathbb{R})}^{m} M_{r}^{+} f(x)\right),
\end{aligned}
$$

where $C>0$ is independent of $x_{0}, h$. Using the arguments similar to those used in deriving the inequality (11) in [20], we get

$$
T_{\epsilon}^{+, b, m} f(y)=T_{\epsilon}^{+}\left(\left(b-b_{I}\right)^{m} f\right)(y)+\sum_{k=0}^{m-1} C_{k, m}\left(b(y)-b_{I}\right)^{m-k} T_{\epsilon}^{+, b, k} f(y), \quad \forall y \in \mathbb{R} .
$$

Note that $T_{\epsilon}^{+, b, k} f_{3}(y)=0$ for any $\epsilon>0,0 \leq k \leq m-1$ and $y \geq x_{0}$. (24) leads to

$$
\begin{aligned}
V\left(\mathcal{T}_{b}^{m}\right) f(y)= & V(\mathcal{T})\left(\left(b-b_{I}\right)^{m} f_{1}\right)(y)+V(\mathcal{T})\left(\left(b-b_{I}\right)^{m} f_{2}\right)(y) \\
& +\sum_{k=0}^{m-1} C_{k, m}\left(b(y)-b_{I}\right)^{m-k} V\left(\mathcal{T}_{b}^{k}\right) f(y), \quad \forall y \geq x_{0} .
\end{aligned}
$$

We notice from (11) that

$$
\begin{aligned}
& \frac{1}{h} \int_{x_{0}}^{x_{0}+h}\left|\mathcal{V}_{\varrho}\left(\mathcal{T}_{b}^{m}\right) f(y)-\mathcal{V}_{\varrho}\left(\mathcal{T}_{b}^{m}\right)\left(\left(b-b_{I}\right)^{m} f_{2}\right)\left(x_{0}\right)\right| d y \\
= & \frac{1}{h} \int_{x_{0}}^{x_{0}+h}\left|\left\|V\left(\mathcal{T}_{b}^{m}\right) f(y)\right\|_{F_{\varrho}}-\left\|V\left(\mathcal{T}_{b}^{m}\right)\left(\left(b-b_{I}\right)^{m} f_{2}\right)\left(x_{0}\right)\right\|_{F_{\varrho}}\right| d y \\
\leq & \frac{1}{h} \int_{x_{0}}^{x_{0}+h}\left\|V\left(\mathcal{T}_{b}^{m}\right) f(y)-V\left(\mathcal{T}_{b}^{m}\right)\left(\left(b-b_{I}\right)^{m} f_{2}\right)\left(x_{0}\right)\right\|_{F_{\varrho}} d y .
\end{aligned}
$$

This together with (25) and (11) yield that

$$
\begin{aligned}
& \frac{1}{h} \int_{x_{0}}^{x_{0}+h}\left|\mathcal{V}_{\varrho}\left(\mathcal{T}_{b}^{m}\right) f(y)-\mathcal{V}_{\varrho}\left(\mathcal{T}_{b}^{m}\right)\left(\left(b-b_{I}\right)^{m} f_{2}\right)\left(x_{0}\right)\right| d y \\
\leq & \frac{1}{h} \int_{x_{0}}^{x_{0}+h} \mathcal{V}_{\varrho}(\mathcal{T})\left(\left(b-b_{I}\right)^{m} f_{1}\right)(y) d y \\
& +\sum_{k=0}^{m-1} C_{k, m} \frac{1}{h} \int_{x_{0}}^{x_{0}+h}\left|b(y)-b_{I}\right|^{m-k} \mathcal{V}_{\varrho}\left(\mathcal{T}_{b}^{k}\right) f(y) d y \\
& +\frac{1}{h} \int_{x_{0}}^{x_{0}+h}\left\|V(\mathcal{T})\left(\left(b-b_{I}\right)^{m} f_{2}\right)(y)-V(\mathcal{T})\left(\left(b-b_{I}\right)^{m} f_{2}\right)\left(x_{0}\right)\right\|_{F_{\varrho}} d y \\
= & : I_{1}+I_{2}+I_{3} .
\end{aligned}
$$

Observe that, for any $\delta>1$ and $k \in \mathbb{N}$,

$$
\begin{aligned}
\frac{1}{\left|2^{k} I\right|} \int_{2^{k} I}\left|b(z)-b_{I}\right|^{\delta} d z & \leq 2^{\delta-1}\left(\frac{1}{\left|2^{k} I\right|} \int_{2^{k} I}\left|b(z)-b_{2^{k} I}\right|^{\delta} d z+\left|b_{I}-b_{2^{k} I}\right|^{\delta}\right) \\
& \leq C(\delta)(k+1)^{\delta}\|b\|_{\mathrm{BMO}(\mathbb{R})}^{\delta}
\end{aligned}
$$


We set $\rho=\sqrt{r}$. By Hölder's inequality, the $L^{\rho}$ boundedness for $\mathcal{V}_{\varrho}(\mathcal{T})$ and (27), we have

$$
\begin{aligned}
I_{1} & \leq\left(\frac{1}{h} \int_{x_{0}}^{x_{0}+h}\left|\mathcal{V}_{\varrho}(\mathcal{T})\left(\left(b-b_{I}\right)^{m} f_{1}\right)(y)\right|^{\rho} d y\right)^{1 / \rho} \\
& \leq C(\rho)\left(\frac{1}{h} \int_{x_{0}}^{x_{0}+2 h}\left|\left(b(y)-b_{I}\right)^{m} f(y)\right|^{\rho} d y\right)^{1 / \rho} \\
& \leq C(\rho)\left(\frac{1}{h} \int_{x_{0}}^{x_{0}+2 h}|f(y)|^{r} d y\right)^{1 / r}\left(\frac{1}{|I|} \int_{I}\left|b(y)-b_{I}\right|^{m \rho \rho^{\prime}} d y\right)^{1 / \rho \rho^{\prime}} \\
& \leq C(m, r)\|b\|_{\mathrm{BMO}(\mathbb{R})}^{m} M_{r}^{+} f\left(x_{0}\right)
\end{aligned}
$$

and

$$
\begin{aligned}
I_{2} & \leq \sum_{k=0}^{m-1} C_{k, m}\left(\frac{1}{h} \int_{x_{0}}^{x_{0}+h}\left|\mathcal{V}_{\varrho}\left(\mathcal{T}_{b}^{k}\right) f(y)\right|^{r} d y\right)^{1 / r}\left(\frac{1}{|I|} \int_{|I|} \mid\left(b(y)-b_{I}\right)^{(m-k) r^{\prime}} d y\right)^{1 / r^{\prime}} \\
& \leq C(m, r) \sum_{k=0}^{m-1} C_{k, m}\|b\|_{\mathrm{BMO}(\mathbb{R})}^{m-k} M_{r}^{+}\left(\mathcal{V}_{\varrho}\left(\mathcal{T}_{b}^{k}\right) f\right)\left(x_{0}\right) .
\end{aligned}
$$

For $I_{3}$, let $y \in\left[x_{0}, x_{0}+h\right]$ and $\beta=\left\{\epsilon_{i}\right\} \in \Theta$, since

$$
\begin{aligned}
& T_{\left[\epsilon_{i+1}, \epsilon_{i}\right]}^{+}\left(\left(b-b_{I}\right)^{m} f_{2}\right)(y)-T_{\left[\epsilon_{i+1}, \epsilon_{i}\right]}^{+}\left(\left(b-b_{I}\right)^{m} f_{2}\right)\left(x_{0}\right) \\
= & \int_{\mathbb{R}}\left[K(y-z) \chi\left(y+\epsilon_{i+1}, y+\epsilon_{i}\right]\right. \\
= & \left.\int_{\mathbb{R}}(z)-K\left(x_{0}-z\right) \chi_{\left(x_{0}+\epsilon_{i+1}, x_{0}+\epsilon_{i}\right]}(z)\right]\left(b(z)-b_{I}\right)^{m} f_{2}(z) d z \\
& +\int_{\mathbb{R}}\left[K\left(x_{0}-z\right)\left(\chi_{\left(y+\epsilon_{i+1}, y+\epsilon_{i}\right]}(z)-\chi_{\left(x_{0}+\epsilon_{i+1}, x_{0}+\epsilon_{i}\right]}(z)\right)\left(b(z)-b_{I}\right)^{m} f_{2}(z) d z .\right.
\end{aligned}
$$

It follows that

$$
\begin{aligned}
& \left\|V(\mathcal{T})\left(\left(b-b_{I}\right)^{m} f_{2}\right)(y)-V(\mathcal{T})\left(\left(b-b_{I}\right)^{m} f_{2}\right)\left(x_{0}\right)\right\|_{F_{Q}} \\
\leq & \|\left\{\int_{\mathbb{R}}\left(K(y-z)-K\left(x_{0}-z\right)\right) \chi\left(y+\epsilon_{i+1}, y+\epsilon_{i}\right]\right. \\
& +\|\left\{\int_{\mathbb{R}} K\left(x_{0}-z\right)\left(\chi_{\left(y+\epsilon_{i+1}, y+\epsilon_{i}\right]}(z)-\chi_{\left(x_{0}+\epsilon_{i+1}, x_{0}+\epsilon_{i}\right]}(z)\right)\right. \\
& \left.\times\left(b(z)-b_{I}\right)^{m} f_{2}(z) d z\right\}_{i \in \mathbb{N}, \beta=\left\{\epsilon_{i}\right\} \in \Theta} \|_{F_{Q}} \\
= & : I_{11}+I_{12} .
\end{aligned}
$$

Since $\left|x_{0}-z\right|>2 h \geq 2\left|x_{0}-y\right|$ for $z>x_{0}+2 h$, then $\left|K(y-z)-K\left(x_{0}-z\right)\right| \leq B_{3}\left|x_{0}-y\right| \mid x_{0}-$ $\left.z\right|^{-2} \leq B_{3} h\left|x_{0}-z\right|^{-2}$ for any $z>x_{0}+2 h$. Note that

$$
\left\|\left\{\chi_{\left(y+\epsilon_{i+1}, y+\epsilon_{i}\right]}(z)\right\}_{i \in \mathbb{N}, \beta=\left\{\epsilon_{i}\right\} \in \Theta}\right\|_{F_{Q}} \leq 1, \forall y \in \mathbb{R} .
$$


By Minkowski's inequality, Hölder's inequality and (27) with $\delta=m r^{\prime}$, we obtain

$$
\begin{aligned}
I_{11} \leq & \int_{\mathbb{R}}\left|K(y-z)-K\left(x_{0}-z\right)\right|\left\|\left\{\chi_{\left(y+\epsilon_{i+1}, y+\epsilon_{i}\right]}(z)\right\}_{i \in \mathbb{N}, \beta=\left\{\epsilon_{i}\right\} \in \Theta}\right\|_{F_{Q}} \\
& \times\left|\left(b(z)-b_{I}\right)^{m} f_{2}(z)\right| d z \\
\leq & B_{3} h \int_{x_{0}+2 h}^{\infty} \frac{\left|\left(b(z)-b_{I}\right)^{m} f(z)\right|}{\left(z-x_{0}\right)^{2}} d z \\
\leq & B_{3} h \sum_{k=1}^{\infty} \int_{x_{0}+2^{k} h}^{x_{0}+2^{k+1} h} \frac{\left|\left(b(z)-b_{I}\right)^{m} f(z)\right|}{\left(2^{k} h\right)^{2}} d z \\
\leq & 4 B_{3} \sum_{k=1}^{\infty} 2^{-k}\left(\frac{1}{2^{k+1} h} \int_{x_{0}}^{x_{0}+2^{k+1} h}|f(z)|^{r} d z\right)^{1 / r}\left(\frac{1}{\left|2^{k} I\right|} \int_{2^{k} I}\left|b(z)-b_{I}\right|^{m r^{\prime}} d z\right)^{1 / r^{\prime}} \\
\leq & 4 B_{3} \sum_{k=1}^{\infty} \frac{(k+1)^{m}}{2^{k}}\|b\|_{\mathrm{BMO}(\mathbb{R})}^{m} M_{r}^{+} f\left(x_{0}\right) \leq C\left(m, r, B_{3}\right)\|b\|_{\mathrm{BMO}(\mathbb{R})}^{m} M_{r}^{+} f\left(x_{0}\right) .
\end{aligned}
$$

It remains to estimate $I_{12}$. Fix $\left\{\epsilon_{i}\right\} \in \Theta$. Let $N_{1}=\left\{i \in \mathbb{Z}: \epsilon_{i}-\epsilon_{i+1} \geq y-x_{0}\right\}$ and $N_{2}=\{i \in \mathbb{Z}$ : $\left.\epsilon_{i}-\epsilon_{i+1}<y-x_{0}\right\}$. We can write

$$
\begin{aligned}
& \sum_{i \in \mathbb{Z}}\left|\int_{\mathbb{R}} K\left(x_{0}-z\right)\left(\chi_{\left(y+\epsilon_{i+1}, y+\epsilon_{i}\right]}(z)-\chi_{\left(x_{0}+\epsilon_{i+1}, x_{0}+\epsilon_{i}\right]}(z)\right)\left(b(z)-b_{I}\right)^{m} f_{2}(z) d z\right|^{\rho} \\
& \leq \sum_{i \in N_{1}}\left|\int_{\mathbb{R}} K\left(x_{0}-z\right)\left(\chi_{\left(y+\epsilon_{i+1}, y+\epsilon_{i}\right]}(z)-\chi_{\left(x_{0}+\epsilon_{i+1}, x_{0}+\epsilon_{i}\right]}(z)\right)\left(b(z)-b_{I}\right)^{m} f_{2}(z) d z\right|^{\rho} \\
& \quad+\sum_{i \in N_{2}}\left|\int_{\mathbb{R}} K\left(x_{0}-z\right)\left(\chi_{\left(y+\epsilon_{i+1}, y+\epsilon_{i}\right]}(z)-\chi_{\left(x_{0}+\epsilon_{i+1}, x_{0}+\epsilon_{i}\right]}(z)\right)\left(b(z)-b_{I}\right)^{m} f_{2}(z) d z\right|^{\rho} \\
& =: J_{11}+J_{12} .
\end{aligned}
$$

By Hölder's inequality, we obtain

$$
\begin{aligned}
J_{11} \leq & B_{2}^{\rho} \sum_{i \in N_{1}}\left|\int_{\mathbb{R}} \frac{\left|\left(b(z)-b_{I}\right)^{m} f_{2}(z)\right|}{\left|x_{0}-z\right|}\left(\chi_{\left(x_{0}+\epsilon_{i+1}, y+\epsilon_{i+1}\right]}(z)+\chi_{\left(x_{0}+\epsilon_{i}, y+\epsilon_{i}\right]}(z)\right) d z\right|^{\rho} \\
\leq & \left(4 B_{2}\right)^{\rho} \sum_{i \in N_{1}}\left|\int_{\mathbb{R}} \frac{\left|\left(b(z)-b_{I}\right)^{m} f_{2}(z)\right|}{\left|x_{0}-z\right|} \chi_{\left(x_{0}+\epsilon_{i}, y+\epsilon_{i}\right]}(z) d z\right|^{\rho} \\
\leq & \left(4 B_{2}\right)^{\rho} h^{\rho-1} \sum_{i \in N_{1}} \int_{\mathbb{R}} \frac{\left|\left(b(z)-b_{I}\right)^{m} f_{2}(z)\right|^{\rho}}{\left|x_{0}-z\right|^{\rho}} \chi_{\left(y+\epsilon_{i+1}, y+\epsilon_{i}\right]}(z) d z \\
\leq & \left(4 B_{2}\right)^{\rho} h^{\rho-1} \int_{\mathbb{R}} \frac{\left|\left(b(z)-b_{I}\right)^{m} f_{2}(z)\right|^{\rho}}{\left|x_{0}-z\right|^{\rho}} d z . \\
J_{12} \leq & B_{2}^{\rho} \sum_{i \in N_{2}}\left|\int_{\mathbb{R}} \frac{\left|\left(b(z)-b_{I}\right)^{m} f_{2}(z)\right|}{\left|x_{0}-z\right|}\left(\chi_{\left(y+\epsilon_{i+1}, y+\epsilon_{i}\right]}(z)+\chi_{\left(x_{0}+\epsilon_{i+1}, x_{0}+\epsilon_{i}\right]}(z)\right) d z\right|^{\rho} \\
\leq & \left(2 B_{2}\right)^{\rho} \sum_{i \in N_{2}} \mid \int_{\mathbb{R}} \frac{\left|\left(b(z)-b_{I}\right)^{m} f_{2}(z)\right|}{\left|x_{0}-z\right|}\left(\left.\chi_{\left(y+\epsilon_{i+1}, y+\epsilon_{i}\right]}(z) d z\right|^{\rho}\right. \\
& +\left(2 B_{2}\right)^{\rho} \sum_{i \in N_{2}}\left|\int_{\mathbb{R}} \frac{\left|\left(b(z)-b_{I}\right)^{m} f_{2}(z)\right|}{\left|x_{0}-z\right|} \chi_{\left(x_{0}+\epsilon_{i+1}, x_{0}+\epsilon_{i}\right]}(z) d z\right|^{\rho} \\
\leq & h^{\rho-1}\left(2 B_{2}\right)^{\rho} \sum_{i \in N_{2}} \int_{\mathbb{R}} \frac{\left|\left(b(z)-b_{I}\right)^{m} f_{2}(z)\right|^{\rho}}{\left|x_{0}-z\right|^{\rho}} \chi_{\left(y+\epsilon_{i+1}, y+\epsilon_{i}\right]}(z) d z \\
& +h^{\rho-1}\left(2 B_{2}\right)^{\rho} \sum_{i \in N_{2}} \int_{\mathbb{R}} \frac{\left|\left(b(z)-b_{I}\right)^{m} f_{2}(z)\right|^{\rho}}{\left|x_{0}-z\right|^{\rho}} \chi_{\left(x_{0}+\epsilon_{i+1}, x_{0}+\epsilon_{i}\right]}(z) d z \\
\leq & 2\left(2 B_{2}\right)^{\rho} h^{\rho-1} \int_{\mathbb{R}} \frac{\left|\left(b(z)-b_{I}\right)^{m} f_{2}(z)\right|^{\rho}}{\left|x_{0}-z\right|^{\rho}} d z . \\
& \\
& \\
&
\end{aligned}
$$

It follows from (32)-(34) that

$$
I_{12} \leq C\left(B_{2}, r\right) h^{1-1 / \rho}\left(\int_{\mathbb{R}} \frac{\left|\left(b(z)-b_{I}\right)^{m} f_{2}(z)\right|^{\rho}}{\left|x_{0}-z\right|^{\rho}} d z\right)^{1 / \rho} .
$$


By Hölder's inequality and (27) (with $\left.\delta=m \rho \rho^{\prime}\right)$, we have

$$
\begin{aligned}
& \int_{\mathbb{R}} \frac{\left|\left(b(z)-b_{I}\right)^{m} f_{2}(z)\right|^{\rho}}{\left|x_{0}-z\right|^{\rho}} d z \\
& =\sum_{k=1}^{\infty} \int_{x_{0}+2^{k} h}^{x_{0}+2^{k+1} h} \frac{\left|\left(b(z)-b_{I}\right)^{m} f(z)\right|^{\rho}}{\left|x_{0}-z\right|^{\rho}} d z \\
& \leq \sum_{k=1}^{\infty}\left(2^{k} h\right)^{-\rho} \int_{x_{0}+2^{k} h}^{x_{0}+2^{k+1} h}\left|\left(b(z)-b_{I}\right)^{m} f(z)\right|^{\rho} d z \\
& \leq 4 h^{1-\rho} \sum_{k=1}^{\infty} 2^{-k(\rho-1)}\left(\frac{1}{2^{k+1} h} \int_{x_{0}}^{x_{0}+2^{k+1} h}|f(z)|^{r} d z\right)^{1 / \rho} \\
& \times\left(\frac{1}{\left|2^{k} I\right|} \int_{2^{k} I}\left|b(z)-b_{I}\right|^{m \rho \rho^{\prime}} d z\right)^{1 / \rho^{\prime}} \\
& \leq 4 h^{1-\rho}\|b\|_{\mathrm{BMO}(\mathbb{R})}^{m \rho} \sum_{k=1}^{\infty} \frac{(k+1)^{m \rho}}{2^{k(\rho-1)}}\left(M_{r}^{+} f\left(x_{0}\right)\right)^{\rho} .
\end{aligned}
$$

This yields directly

$$
\int_{\mathbb{R}} \frac{\left|\left(b(z)-b_{I}\right)^{m} f_{2}(z)\right|^{\rho}}{\left|x_{0}-z\right|^{\rho}} d z \leq C(m, r) h^{1-\rho}\|b\|_{\mathrm{BMO}(\mathbb{R})}^{m \rho}\left(M_{r}^{+} f\left(x_{0}\right)\right)^{\rho} .
$$

Combining (36) with (35) yields (37) together with (30) and (31) implies

$$
\begin{gathered}
I_{12} \leq C\left(m, r, B_{2}\right)\|b\|_{\mathrm{BMO}(\mathbb{R})}^{m} M_{r}^{+} f\left(x_{0}\right), \\
I_{3} \leq C\left(m, r, B_{2}, B_{3}\right)\|b\|_{\mathrm{BMO}(\mathbb{R})}^{m} M_{r}^{+} f\left(x_{0}\right) .
\end{gathered}
$$

Combining (38) with (26), (28) and (29) yields (23). This completes the proof.

We now turn to prove our main results.

Proof of Theorem 1. We first prove (i). For any $w \in A_{p}^{+}$with $1<p<\infty$, there exists $r \in(1, p)$ such that $w \in A_{p / r}^{+}$. Then, we have

$$
\left\|M_{r}^{+} f\right\|_{L^{p}(\mathbb{R}, w(x) d x)} \leq\left\|M^{+}|f|^{r}\right\|_{L^{p / r}(\mathbb{R}, w(x) d x)}^{1 / r} \leq C_{p, r}\|f\|_{L^{p}(\mathbb{R}, w(x) d x)} .
$$

On the other hand, it was proved in [23] that

$$
\left\|M^{+} f\right\|_{L^{p}(\mathbb{R}, w(x) d x)} \leq C\left\|M^{+, \sharp} f\right\|_{L^{p}(\mathbb{R}, w(x) d x)}
$$

for $1<p<\infty$ and $w \in A_{\infty}^{+}$. We get from (22), (39) and (40) and that

$$
\begin{aligned}
\left\|\mathcal{V}_{\varrho}(\mathcal{T}) f\right\|_{L^{p}(\mathbb{R}, w(x) d x)} & \leq\left\|M^{+}\left(\mathcal{V}_{\varrho}(\mathcal{T}) f\right)\right\|_{L^{p}(\mathbb{R}, w(x) d x)} \\
& \left.\leq C \| M^{+, \sharp}\left(\mathcal{V}_{\varrho}(\mathcal{T}) f\right)\right) \|_{L^{p}(\mathbb{R}, w(x) d x)} \\
& \leq C\left\|M_{r}^{+} f\right\|_{L^{p}(\mathbb{R}, w(x) d x)} \leq C\|f\|_{L^{p}(\mathbb{R}, w(x) d x)} .
\end{aligned}
$$

This together with Lemma 3 yields Theorem 1 (i).

Applying Lemma 4 and the arguments similar to those used in deriving Theorem 1.3 in [19], we can get Theorem 1 (ii). The details are omitted.

We now prove (iii). For $w^{-1} \in A_{1}^{-}$, there exists $r>1$ such that $w^{-r} \in A_{1}^{-}$. Thus, for any $x \in \mathbb{R}$,

$$
\begin{aligned}
M_{r}^{+} f(x) w(x) & =w(x)\left(\sup _{h>0} \frac{1}{h} \int_{x}^{x+h}(|f(y)| w(y))^{r} w^{-r}(y) d y\right)^{1 / r} \\
& \leq\|f\|_{L^{\infty}(\mathbb{R}, w(x) d x)} w(x)\left(M^{+}\left(w^{-r}\right)(x)\right)^{1 / r} \leq\left\|w^{-r}\right\|_{A_{1}^{-}}\|f\|_{L^{\infty}(\mathbb{R}, w(x) d x)},
\end{aligned}
$$


which together with (23) yield that

$$
\begin{aligned}
\left\|\mathcal{V}_{\varrho}(\mathcal{T}) f\right\|_{\mathrm{BMO}^{+}(\mathbb{R}, w(x) d x)} & \left.=\| M^{+, \sharp}\left(\mathcal{V}_{\varrho}(\mathcal{T}) f\right)\right) \|_{L^{\infty}(\mathbb{R}, w(x) d x)} \\
& \leq C\left\|M_{r}^{+} f\right\|_{L^{\infty}(\mathbb{R}, w(x) d x)} \leq C\|f\|_{L^{\infty}(\mathbb{R}, w(x) d x)}
\end{aligned}
$$

for any $1<r<\infty$. This proves Theorem 1 .

Proof of Theorem 2. We first prove (i). Fix $x_{0} \in \mathbb{R}$ and $h>0$. It suffices to show that

$$
\left(\frac{1}{w\left(x_{0}-h, x_{0}\right)} \int_{x_{0}}^{x_{0}+h}\left|\mathcal{V}_{\varrho}\left(\mathcal{T}_{b}^{m}\right) f(x)\right|^{p} d x\right)^{1 / p} \leq C\|b\|_{\mathrm{BMO}(\mathbb{R})}^{m} h^{\beta}\|f\|_{L^{p, \beta,+}(w)^{\prime}}
$$

where $C>0$ is independent of $x_{0}, h$. Let $f_{1}=f \chi_{\left[x_{0}, x_{0}+2 h\right)}, f_{2}=f \chi_{\left[x_{0}+2 h, \infty\right)}$ and $f_{3}=f-f_{1}-f_{2}$. Let $I=\left[x_{0}-2 h, x_{0}+2 h\right]$. Note that $T_{\epsilon}^{+, b, m} f_{3}(x)=0$ for any $\epsilon>0$ and $x \geq x_{0}$. It follows that $\mathcal{V}_{\varrho}\left(\mathcal{T}_{b}^{m}\right) f_{3}(x)=0$ for all $x \geq x_{0}$. Thus, we can write

$$
\begin{aligned}
& \left(\frac{1}{w\left(x_{0}-h, x_{0}\right)} \int_{x_{0}}^{x_{0}+h}\left|\mathcal{V}_{\varrho}\left(\mathcal{T}_{b}^{m}\right) f(x)\right|^{p} d x\right)^{1 / p} \\
\leq & \left(\frac{1}{w\left(x_{0}-h, x_{0}\right)} \int_{x_{0}}^{x_{0}+h}\left|\mathcal{V}_{\varrho}\left(\mathcal{T}_{b}^{m}\right) f_{1}(x)\right|^{p} d x\right)^{1 / p} \\
& +\left(\frac{1}{w\left(x_{0}-h, x_{0}\right)} \int_{x_{0}}^{x_{0}+h}\left|\mathcal{V}_{\varrho}\left(\mathcal{T}_{b}^{m}\right) f_{2}(x)\right|^{p} d x\right)^{1 / p}=: S_{1}+S_{2} .
\end{aligned}
$$

Invoking Lemma 1 (i) and Theorem 1 (ii), there exists $C>0$ independent of $x_{0}, h$, such that

$$
\begin{aligned}
S_{1} & \leq C\|b\|_{\mathrm{BMO}(\mathbb{R})}^{m}\left(\frac{1}{w\left(x_{0}-h, x_{0}\right)} \int_{x_{0}}^{x_{0}+2 h}|f(x)|^{p} d x\right)^{1 / p} \\
& \leq C\|b\|_{\mathrm{BMO}(\mathbb{R})}^{m}\left(\frac{w\left(x_{0}-2 h, x_{0}\right)}{w\left(x_{0}-h, x_{0}\right)} \frac{1}{w\left(x_{0}-2 h, x_{0}\right)} \int_{x_{0}}^{x_{0}+2 h}|f(x)|^{p} d x\right)^{1 / p} \\
& \leq C\|b\|_{\mathrm{BMO}(\mathbb{R})}^{m} h^{\beta}\|f\|_{L^{p, \beta,+}(w)} .
\end{aligned}
$$

Applying Lemma 1 (ii), there exists $C>0$ independent of $x_{0}, h$ such that

$$
\begin{aligned}
& \left(\frac{1}{w\left(x_{0}-h, x_{0}\right)} \int_{x_{0}+2^{k} h}^{x_{0}+2^{k+1} h}|f(z)|^{p} d z\right)^{1 / p} \\
\leq & \left(\frac{w\left(x_{0}-h-2^{k+2} h, x_{0}-h\right)}{w\left(x_{0}-h, x_{0}\right)} \frac{1}{w\left(x_{0}-h-2^{k+2} h, x_{0}-h\right)} \int_{x_{0}-h}^{x_{0}-h+2^{k+2} h}|f(z)|^{p} d z\right)^{1 / p} \\
\leq & C 2^{(k+2)(1+\beta)} h^{\beta}\|f\|_{L^{p, \beta,+}(w)} .
\end{aligned}
$$

One can easily check that $|x-z|>\left|z-x_{0}\right| / 2$ for $x \in\left[x_{0}, x_{0}+h\right]$ and $z \in\left[x_{0}+2 h, \infty\right)$. Fix $x \in$ $\left[x_{0}, x_{0}+h\right]$. Then, by (11) and Minkowski's inequality, we have

$$
\begin{aligned}
\mathcal{V}_{\varrho}\left(\mathcal{T}_{b}^{m}\right) f_{2}(x) & =\left\|V\left(\mathcal{T}_{b}^{m}\right) f_{2}(x)\right\|_{F_{Q}} \\
& \leq\left\|\left\{\int_{\epsilon_{i+1}<z-x \leq \epsilon_{i}} K(x-z)(b(x)-b(z))^{m} f_{2}(z) d z\right\}_{i \in \mathbb{N}, \beta=\left\{\epsilon_{i}\right\} \in \Theta}\right\|_{F_{\varrho}} \\
& \leq \int_{\mathbb{R}}\left|K(x-z)(b(x)-b(z))^{m} f_{2}(z)\right|\left\|\left\{\chi_{\epsilon_{i+1}<z-x \leq \epsilon_{i}}\right\}_{i \in \mathbb{N}, \beta=\left\{\epsilon_{i}\right\} \in \Theta}\right\|_{F_{\varrho}} d z \\
& \leq C \int_{\mathbb{R}} \frac{\left|f_{2}(z)(b(x)-b(z))^{m}\right|}{\left|z-x_{0}\right|} d z,
\end{aligned}
$$


where $C>0$ is independent of $x_{0}, h$. It is clear that

$$
\int_{\mathbb{R}} \frac{\left|f_{2}(z)(b(x)-b(z))^{m}\right|}{\left|z-x_{0}\right|} d z=\sum_{k=1}^{\infty} \int_{x_{0}+2^{k} h}^{x_{0}+2^{k+1} h} \frac{\left|f(z)(b(x)-b(z))^{m}\right|}{\left|z-x_{0}\right|} d z .
$$

Fix $k \geq 1$. By Hölder's inequality, we obtain

$$
\begin{aligned}
& \int_{x_{0}+2^{k} h}^{x_{0}+2^{k+1} h} \frac{\left|f(z)(b(x)-b(z))^{m}\right|}{\left|z-x_{0}\right|} d z \\
\leq & 2^{m}\left(2^{k} h\right)^{-1}\left(\int_{x_{0}+2^{k} h}^{x_{0}+2^{k+1} h}|f(z)|\left|b(x)-b_{2^{k}} I^{m} d z+\int_{x_{0}+2^{k} h}^{x_{0}+2^{k+1} h}\right| f(z)|| b(z)-\left.b_{2^{k} I}\right|^{m} d z\right) \\
\leq & 2^{m}\left(2^{k} h\right)^{-1 / p}\left|b(x)-b_{2^{k} I}\right|^{m}\left(\int_{x_{0}+2^{k} h}^{x_{0}+2^{k+1} h}|f(z)|^{p} d z\right)^{1 / p} \\
& +2^{m}\left(2^{k} h\right)^{-1}\left(\int_{x_{0}+2^{k} h}^{x_{0}+2^{k+1} h}|f(z)|^{p} d z\right)^{1 / p}\left(\int_{x_{0}+2^{k} h}^{x_{0}+2^{k+1} h}\left|b(z)-b_{2^{k} I}\right|^{m p^{\prime}} d z\right)^{1 / p^{\prime}} .
\end{aligned}
$$

This together with (27) and (44) yields that

$$
\begin{aligned}
& \int_{x_{0}+2^{k h}}^{x_{0}+2^{k+1} h} \frac{\left|f(z)(b(x)-b(z))^{m}\right|}{\left|z-x_{0}\right|} d z \\
\leq & C 2^{k(1+\beta)} h^{\beta}\|f\|_{L^{p, \beta,+}(w)}\left(2^{k} h\right)^{-1 / p} w\left(\left(x_{0}-h, x_{0}\right)\right)^{1 / p}\left(\left|b(x)-b_{2^{k} I}\right|^{m}+\|b\|_{\mathrm{BMO}(\mathbb{R})}^{m}\right) .
\end{aligned}
$$

Here, $C>0$ is independent of $x_{0}, h$. By (45) and (46) and Hölder's inequality, we have

$$
\begin{aligned}
S_{2} \leq & C h^{\beta}\|f\|_{L^{p, \beta,+}(w)} \sum_{k=1}^{\infty} 2^{k(1+\beta)}\left(2^{k} h\right)^{-1 / p} \\
& \times\left(\int_{x_{0}}^{x_{0}+h}\left|\left(\left|b(x)-b_{2^{k} I}\right|^{m}+\|b\|_{\mathrm{BMO}(\mathbb{R})}^{m}\right)\right|^{p} d x\right)^{1 / p} \\
\leq & C h^{\beta}\|f\|_{L^{p, \beta,+}(w)} \sum_{k=1}^{\infty} 2^{k(1+\beta)}\left(2^{k} h\right)^{-1 / p} \\
& \times\left(\int_{x_{0}}^{x_{0}+h}\left(2^{m}\left|b(x)-b_{I}\right|^{m}+2^{m}\left|b_{I}-b_{2^{k} I}\right|^{m}+\|b\|_{\mathrm{BMO}(\mathbb{R})}^{m}\right)^{p} d x\right)^{1 / p} \\
\leq & C\|b\|_{\mathrm{BMO}(\mathbb{R})}^{m} h^{\beta}\|f\|_{L^{p, \beta,+}(w)} \sum_{k=1}^{\infty} \frac{(k+1)^{m}}{2^{(1 / p-1-\beta) k}} \\
\leq & C\|b\|_{\mathrm{BMO}(\mathbb{R})}^{m} h^{\beta}\|f\|_{L^{p, \beta,+}(w)} .
\end{aligned}
$$

Here, $C>0$ is independent of $x_{0}, h$. In the last inequality of (47), we have used the condition $1 / p>1+\beta$. (47) together with (42) and (43) yield (41).

Next, we prove (ii). Let $f_{1}=f \chi_{\left[x_{0}, x_{0}+2 h\right)}, f_{2}=f \chi_{\left[x_{0}+2 h, \infty\right)}$ and $f_{3}=f-f_{1}-f_{2}$. Let $I=$ $\left[x_{0}-2 h, x_{0}+2 h\right]$. By (4), we want to show that

$$
\left(\frac{1}{w\left(x_{0}-h, x_{0}\right)} \int_{x_{0}}^{x_{0}+h}\left|\mathcal{V}_{\varrho}(\mathcal{T}) f(x)-\mathcal{V}_{\varrho}(\mathcal{T}) f_{2}\left(x_{0}\right)\right|^{p} d x\right)^{1 / p} \leq C h^{\beta}\|f\|_{L^{p, \beta,+}(w)},
$$

where $C>0$ independent of $x_{0}, h$. Using (11) and Minkowski's inequality, one has

$$
\begin{aligned}
& \left|\mathcal{V}_{\varrho}(\mathcal{T}) f(x)-\mathcal{V}_{\varrho}(\mathcal{T}) f_{2}\left(x_{0}\right)\right| \\
= & \left|\|V(\mathcal{T}) f(x)\|_{F_{\varrho}}-\left\|V(\mathcal{T}) f_{2}\left(x_{0}\right)\right\|_{F_{\varrho}}\right| \\
\leq & \left\|V(\mathcal{T}) f(x)-V(\mathcal{T}) f_{2}\left(x_{0}\right)\right\|_{F_{\varrho}} \leq\left|\mathcal{V}_{\varrho}(\mathcal{T}) f_{1}(x)\right|+\left\|V(\mathcal{T}) f_{2}(x)-V(\mathcal{T}) f_{2}\left(x_{0}\right)\right\|_{F_{\varrho}} .
\end{aligned}
$$


This together with Minkowski's inequality again yield that

$$
\begin{aligned}
& \left(\frac{1}{w\left(x_{0}-h, x_{0}\right)} \int_{x_{0}}^{x_{0}+h}\left|\mathcal{V}_{\varrho}(\mathcal{T}) f(x)-\mathcal{V}_{\varrho}(\mathcal{T}) f_{2}\left(x_{0}\right)\right|^{p} d x\right)^{1 / p} \\
\leq & \left(\frac{1}{w\left(x_{0}-h, x_{0}\right)} \int_{x_{0}}^{x_{0}+h}\left|\mathcal{V}_{\varrho}(\mathcal{T}) f_{1}(x)\right|^{p} d x\right)^{1 / p} \\
& +\left(\frac{1}{w\left(x_{0}-h, x_{0}\right)} \int_{x_{0}}^{x_{0}+h}\left\|V(\mathcal{T}) f_{2}(x)-V(\mathcal{T}) f_{2}\left(x_{0}\right)\right\|_{F_{\varrho}}^{p} d x\right)^{1 / p} .
\end{aligned}
$$

We get from (43) (with $m=0$ ) that

$$
\left(\frac{1}{w\left(x_{0}-h, x_{0}\right)} \int_{x_{0}}^{x_{0}+h}\left|\mathcal{V}_{\varrho}(\mathcal{T}) f_{1}(x)\right|^{p} d x\right)^{1 / p} \leq C h^{\beta}\|f\|_{L^{p, \beta,+}(w)}
$$

where $C>0$ is independent of $x_{0}, h$. Fix $x \in\left[x_{0}, x_{0}+h\right]$. (30), (31) and (35) (with $m=0$ ) imply that

$$
\begin{aligned}
& \left\|V(\mathcal{T}) f_{2}(x)-V(\mathcal{T}) f_{2}\left(x_{0}\right)\right\|_{F_{Q}} \\
\leq & B_{3} h \int_{\mathbb{R}} \frac{\left|f_{2}(z)\right|}{\left|z-x_{0}\right|^{2}} d z+C\left(B_{2}, p\right) h^{1-1 / p}\left(\int_{\mathbb{R}} \frac{\left|f_{2}(z)\right|^{p}}{\left|x_{0}-z\right|^{p}} d z\right)^{1 / p} .
\end{aligned}
$$

It follows that

$$
\begin{aligned}
& \left(\frac{1}{w\left(x_{0}-h, x_{0}\right)} \int_{x_{0}}^{x_{0}+h}\left\|V(\mathcal{T}) f_{2}(x)-V(\mathcal{T}) f_{2}\left(x_{0}\right)\right\|_{F_{Q}}^{p} d x\right)^{1 / p} \\
\leq & \frac{h^{1+1 / p}}{w\left(x_{0}-h, x_{0}\right)^{1 / p}} \int_{x_{0}+2 h}^{\infty} \frac{|f(z)|}{\left(z-x_{0}\right)^{2}} d z+h\left(\frac{1}{w\left(x_{0}-h, x_{0}\right)} \int_{\mathbb{R}} \frac{\left|f_{2}(z)\right|^{p}}{\left|x_{0}-z\right|^{p}} d z\right)^{1 / p} \\
= & : V_{1}+V_{2} .
\end{aligned}
$$

By (44) and Hölder's inequality, there exists $C>0$ independent of $x_{0}, h$, such that

$$
\begin{aligned}
V_{1} & \leq \frac{h^{1+1 / p}}{w\left(x_{0}-h, x_{0}\right)^{1 / p}} \sum_{k=1}^{\infty}\left(2^{k} h\right)^{-2} \int_{x_{0}+2^{k} h}^{x_{0}+2^{k+1} h}|f(z)| d z \\
& \leq \sum_{k=1}^{\infty} 2^{k\left(-2+1 / p^{\prime}\right)}\left(\frac{1}{w\left(x_{0}-h, x_{0}\right)} \int_{x_{0}+2^{k} h}^{x_{0}+2^{k+1} h}|f(z)|^{p} d z\right)^{1 / p} \\
& \leq C \sum_{k=1}^{\infty} 2^{k\left(-2+1 / p^{\prime}\right)} 2^{k(1+\beta)} h^{\beta}\|f\|_{L^{p, \beta,+}(w)} \\
& \leq C \sum_{k=1}^{\infty} 2^{k(\beta-1 / p)} h^{\beta}\|f\|_{L^{p, \beta,+}(w)} \leq C h^{\beta}\|f\|_{L^{p, \beta,+}(w)} . \\
V_{2} & \leq\left(\sum_{k=1}^{\infty} 2^{-k p} \frac{1}{w\left(x_{0}-h, x_{0}\right)} \int_{x_{0}+2^{k} h}^{x_{0}+2^{k+1} h} \frac{|f(z)|^{p}}{\left(z-x_{0}\right)^{p}} d z\right)^{1 / p} \\
& \leq C\left(\sum_{k=1}^{\infty} 2^{-k p} 2^{k(1+\beta) p} h^{\beta p}\|f\|_{L^{p, \beta,+}(w)}^{p}\right)^{1 / p} \\
& \leq C\left(\sum_{k=1}^{\infty} 2^{k \beta p}\right)^{1 / p} h^{\beta}\|f\|_{L^{p, \beta,+}(w)} \leq C h^{\beta}\|f\|_{L^{p, \beta,+}(w)} .
\end{aligned}
$$

(53) together with (49)-(52) yields (48). This finishes the proof of Theorem 2.

\section{4. $\lambda$-Jump Operators and the Number of Up-Crossing}

This section is devoted to study the $\lambda$-jump operators and the number of up-crossing associated with the operators sequence $\left\{T_{\epsilon}^{+, b, m}\right\}_{\epsilon>0}$, which give certain quantitative information on the convergence of the above families of operators. 
Definition 4. Given a family of bounded operators $\mathcal{T}=\left\{T_{\epsilon}\right\}_{\epsilon>0}$ acting between spaces of functions, the $\lambda$-jump operator associated with $\mathcal{T}$ applied to a function $f$ at a point $x$ is defined by

$$
\begin{aligned}
\Lambda_{\lambda}(\mathcal{T}) f(x):= & \sup \left\{n: \text { there exist } s_{1}<t_{1} \leq s_{2}<t_{2}<\cdots \leq s_{n}<t_{n}\right. \\
& \text { such that } \left.\left|T_{s_{i}} f(x)-T_{t_{i}} f(x)\right|>\lambda\right\} .
\end{aligned}
$$

For $0<\alpha<\gamma$, the number of up-crossing associated with $\mathcal{T}$ applied to a function $f$ at a point $x$ is defined by

$$
\begin{aligned}
N_{\alpha, \gamma}(\mathcal{T}) f(x):= & \sup \left\{n: \text { there exist } s_{1}<t_{1}<s_{2}<t_{2}<\ldots<s_{n}<t_{n}\right. \\
& \text { such that } \left.T_{s_{i}} f(x)<\alpha, T_{t_{i}} f(x)>\gamma\right\} .
\end{aligned}
$$

It was shown in [11] that, if the $\lambda$-jump operators is finite a.e. for each choice of $\lambda>0$, then we must have a.e. convergence of our family of operators. Moreover,

$$
\lambda\left(\Lambda_{\lambda}(\mathcal{T}) f(x)\right)^{1 / \varrho} \leq \mathcal{V}_{\varrho}(\mathcal{T}) f(x) \text { and } N_{\alpha, \lambda}(\mathcal{T}) f(x) \leq \Lambda_{\lambda-\alpha}(\mathcal{T}) f(x), \forall \lambda>\alpha>0
$$

By Theorem 1 (ii) and Theorem 2 and (54), we can get the following result.

Theorem 3. Let $m \in \mathbb{N}, \varrho>2, b \in \mathrm{BMO}(\mathbb{R})$ and $K \in \operatorname{OCZK}\left(B_{1}, B_{2}, B_{3}\right)$ with support in $(-\infty, 0)$. Let $\mathcal{T}_{b}^{m}=\left\{T_{\epsilon}^{+, b, m}\right\}_{\epsilon>0}$ and $\mathcal{T}=\left\{T_{\epsilon}^{+}\right\}_{\epsilon>0}$ be given as in (1.1) and (1.2), respectively. Let $\lambda>\alpha>0$. Assume that $\left\|\mathcal{V}_{\varrho}(\mathcal{T})\right\|_{L^{q}(\mathbb{R}, d x) \rightarrow L^{q}(\mathbb{R}, d x)}<\infty$ for some $q \in(1, \infty)$. Then,

(i) for any $1<p<\infty, w \in A_{p}^{+}$and $f \in L^{p}(\mathbb{R}, w(x) d x)$,

$$
\begin{aligned}
\left\|\left(\Lambda_{\lambda}\left(\mathcal{T}_{b}^{m}\right) f\right)^{1 / \varrho}\right\|_{L^{p}(\mathbb{R}, w(x) d x)} & \leq \frac{C(p, \varrho)}{\lambda}\|b\|_{\mathrm{BMO}(\mathbb{R})}^{m}\|f\|_{L^{p}(\mathbb{R}, w(x) d x)} \\
\left\|\left(N_{\alpha, \lambda}\left(\mathcal{T}_{b}^{m}\right) f\right)^{1 / \varrho}\right\|_{L^{p}(\mathbb{R}, w(x) d x)} & \leq \frac{C(p, \varrho)}{\lambda-\alpha}\|b\|_{\mathrm{BMO}(\mathbb{R})}^{m}\|f\|_{L^{p}(\mathbb{R}, w(x) d x)} ;
\end{aligned}
$$

(ii) for any $1<p<1 /(\beta+1),-1 / p \leq \beta<0, w \in A_{p}^{+}$and $f \in L^{p, \beta,+}(w)$,

$$
\begin{gathered}
\left\|\left(\Lambda_{\lambda}\left(\mathcal{T}_{b}^{m}\right) f\right)^{1 / \varrho}\right\|_{L^{p, \beta,+}(w)} \leq \frac{C(p, \varrho)}{\lambda}\|b\|_{\mathrm{BMO}(\mathbb{R})}^{m}\|f\|_{L^{p, \beta,+}(w)} \\
\left\|\left(N_{\alpha, \lambda}\left(\mathcal{T}_{b}^{m}\right) f\right)^{1 / \varrho}\right\|_{L^{p, \beta,+}(w)} \leq \frac{C(p, \varrho)}{\lambda-\alpha}\|b\|_{\mathrm{BMO}(\mathbb{R})}^{m}\|f\|_{L^{p, \beta,+}(w)} .
\end{gathered}
$$

\section{Conclusions and Further Comments}

It should be pointed out that our main results represent one-sided extensions of the main results in $[19,28]$. Combining with the two-sided case, the one-sided case is often more complex. Our main results not only enrich the variation inequalities for singular integrals and related commutators, but also explore some one-sided techniques to serve our aim (for example, see Lemma 1). In fact, it is unknown whether the variation operators for one-sided singular integrals are bounded on $L^{p}(\mathbb{R})$, which will be our forthcoming objective of research. On the other hand, some new one-sided methods and techniques can be explored to apply other one-sided operators.

Author Contributions: Formal analysis, Z.F.; writing-original draft preparation, F.L.; writing-review and editing, S.J. and S.-K.O.

Funding: This work was funded by the NNSF of China (grant Nos.11701333,11671185) and the SP-OYSTTT-CMSS (grant No. Sxy2016K01).

Conflicts of Interest: All of authors in this article declare no conflict of interest. All of funders in this article support the article's publication. 


\section{References}

1. Aimar, H.; Forzani, L.; Martín-Reyes, F.J. On weighted inequalities for singular integrals. Proc. Am. Math. Soc. 1997, 125, 2057-2064. [CrossRef]

2. Bourgain, J. Pointwise ergodic theorems for arithmetric sets. Inst. Hautes Études Sci. Publ. Math. 1989, 69, 5-45. [CrossRef]

3. Campbell, J.T.; Jones, R.L.; Reinhdd, K.; Wierdl, M. Oscillation and variation for the Hilbert transform. Duke Math. J. 2000, 105, 59-83.

4. Campbell, J.T.; Jones, R.L.; Reinhdd, K.; Wierdl, M. Oscillation and variation for singular integrals in higher dimensions. Trans. Am. Math. Soc. 2003, 355, 2115-2137. [CrossRef]

5. Crescimbeni, R.; Martín-Reyes, F.J.; Torre, A.L.; Torrea, J.L. The $\rho$-variation of the Hermitian Riesz transform. Acta Math. Sin. Engl. Ser. 2010, 26, 1827-1838. [CrossRef]

6. Ding, Y.; Hong, G.; Liu, H. Jump and variational inequalities for rough operators. J. Fourier Anal. Appl. 2017, 23, 679-711. [CrossRef]

7. Fu, Z.; Lu, S.; Sato, S.; Shi, S. On weighted weak type norm inequalities for one-sided oscillatory singular integrals. Stud. Math. 2011, 207, 137-150. [CrossRef]

8. Fu, Z.; Lu, S.; Shi, S.; Pan, Y. Some one-sided estimates for oscillatory singular integrals. Nonlinear Anal. 2014, 108, 144-160. [CrossRef]

9. Gillespie, T.A.; Torrea, J.L. Dimension free estimates for the oscillation of Riesz transforms. Israel J. Math. 2004, 141, 125-144. [CrossRef]

10. Harboure, E.; Macías, R.A.; Menárguez, M.T.; Torrea, J.L. Oscillation and variation for the Gaussian Riesz transforms and Poisson integral. Proc. R. Soc. Edinb. 2005, 135A, 85-104. [CrossRef]

11. Jones, R.L. Variation inequalities for singular integrals and related operators. Contemp. Math. 2006, 411, 89-121.

12. Jones, R.L.; Kaufman, R.; Rosenblatt, J.; Wierdl, M. Oscillation in ergodic theory. Ergod. Theory Dyn. Syst. 1998, 18, 889-935. [CrossRef]

13. Jones, R.L.; Rosenblatt, J.; Wierdl, M. Oscillation in ergodic theory: Higher dimensional results. Israel J. Math. 2003, 135, 1-27. [CrossRef]

14. Jones, R.L.; Seeger, A.; Wright, J. Strong variational and jump inequalities in harmonic analysis. Trans. Am. Math. Soc. 2008, 360, 6711-6742. [CrossRef]

15. Lépingle, D. La variation d'ordre $\mathrm{p}$ des semi-martingales. Zeitschrift für Wahrscheinlichkeitstheori Verwandte Gebiete 1976, 36, 295-316. [CrossRef]

16. Liu, F. On the regularity of one-sided fractional maximal functions. Math. Slovaca 2018, 68, 1097-1112. [CrossRef]

17. Liu, F.; Mao, S. On the regularity of the one-sided Hardy-Littlewood functions. Czechoslov. Math. J. 2017, 67, 219-234. [CrossRef]

18. Liu, F.; Xu, L. Regularity of one-sided multilinear fractional maximal functions. Open Math. 2018, 16, 1556-1572. [CrossRef]

19. Liu, F.; Wu, H. A criterion on oscillation and variation for the commutators of singular integral operators. Forum Math. 2015, 27, 77-97. [CrossRef]

20. Lorente, M.; Riveros, M.S. Weighted inequalities for commutators of one-sided singular integrals. Comment. Math. Univ. Carolinae 2002, 43, 83-101.

21. Ma, T.; Torrea, J.L.; Xu, Q. Weighted variation inequalities for differential operators and singular integrals. J. Funct. Anal. 2015, 268, 376-416. [CrossRef]

22. Ma, T.; Torrea, J.L.; Xu, Q. Weighted variation inequalities for differential operators and singular integrals in higher dimensions. Sci. China Math. 2017, 60, 1419-1442. [CrossRef]

23. Martín-Reyes, F.J.; de la Torre, A. One-sided BMO spaces. J. Lond. Math. Soc. 1994, 49, 529-542. [CrossRef]

24. Le Merdy, C.; Xu, Q. Strong $q$-variation inequalities for analytic semigroups. Ann. Inst. Fourier 2012, 62, 2069-2097. [CrossRef]

25. Pisier, G.; Xu, Q. The strong $p$-variation of martingales and orthogonal series. Probab. Theory Relat. Fields 1988, 77, 497-514. [CrossRef]

26. Sawyer, E. Weighted inequalities for the one-sided Hardy-Littlewood maximal functions. Trans. Am. Math. Soc. 1986, 291, 53-61. [CrossRef] 
27. Shi, S.G.; Fu, Z.W. Estimates of some operators on one-sided weighted Morrey spaces. Abstr. Appl. Anal. 2013, 2013, 829218. [CrossRef]

28. Zhang, J.; $\mathrm{Wu}, \mathrm{H}$. Oscillation and variation inequalities for singular integrals and commutators on weighted Morrey spaces. Front. Math. China 2016, 11, 423-447. [CrossRef]

(C) 2019 by the authors. Licensee MDPI, Basel, Switzerland. This article is an open access article distributed under the terms and conditions of the Creative Commons Attribution (CC BY) license (http:/ / creativecommons.org/licenses/by/4.0/). 